JOURNAL OF THE

AMERICAN MATHEMATICAL SOCIETY

Volume 13, Number 4, Pages 971-1009

S 0894-0347(00)00344-1

Article electronically published on June 23, 2000

\title{
TOPOLOGY OF SYMPLECTOMORPHISM GROUPS OF RATIONAL RULED SURFACES
}

\author{
MIGUEL ABREU AND DUSA MCDUFF
}

\section{Contents}

1. Introduction

2. The stratification of $\mathcal{J}_{\lambda}^{i}$ and the groups $K_{k}^{i}$

2.1. Structure of $J$-holomorphic spheres on $M_{\lambda}^{i} \quad 974$

2.2. The strata of $\mathcal{J}_{\lambda}^{i} \quad 975$

2.3. The manifolds $M_{\lambda}^{i}$ as symplectic reductions of $\mathbb{C}^{4} \quad 977$

2.4. Geometric description of the strata $\quad 979$

2.5. Relations between the $K_{k}^{i} \quad 980$

3. Algebraic computations $\quad 983$

3.1. Computation of $H^{*}\left(G_{\lambda}^{0}\right) \quad 984$

3.2. Computation of $H^{*}\left(G_{\lambda}^{1}\right)$

4. The limit at infinity $\quad 996$

5. Relation with Kronheimer's work

6. Whitehead products and $H^{*}\left(B G_{\lambda}\right) \quad 1003$

\begin{tabular}{ll} 
Acknowledgements & 1008 \\
\hline
\end{tabular}

\begin{tabular}{ll} 
References & 1008 \\
\hline
\end{tabular}

\section{INTRODUCTION}

Rational ruled surfaces occur as projectivizations $\mathbf{P}\left(L_{k} \oplus \mathbb{C}\right)$, where $L_{k}$ is a complex line bundle over $S^{2}$ with first Chern class $k$. There are two cases to consider, first ( $k$ is even) when the underlying manifold is diffeomorphic to the product $S^{2} \times S^{2}$ and second ( $k$ is odd) when it is diffeomorphic to $\mathbb{C} P^{2} \# \overline{\mathbb{C}}^{2}$, the one point blow up of $\mathbb{C} P^{2}$. Work of Taubes, Liu-Li and Lalonde-McDuff (for detailed references see [10]) implies that every symplectic form on one of these manifolds is "standard", i.e. it belongs to one of the two following families:

$$
M_{\lambda}^{0}=\left(S^{2} \times S^{2}, \omega_{\lambda}^{0}=(1+\lambda) \sigma_{0} \times \sigma_{0}\right), \quad 0 \leq \lambda \in \mathbb{R},
$$

Received by the editors October 25, 1999 and, in revised form, May 13, 2000.

2000 Mathematics Subject Classification. Primary 57S05, 57R17; Secondary 53D35.

The first author was partially supported by NSF grant DMS 9304580, while at the Institute for Advanced Study (1996/97), and afterwards by FCT grant PCEX/C/MAT/44/96 and PRAXIS XXI through the Research Units Pluriannual Funding Program.

The second author was partially supported by NSF grant DMS 9704825 . 
where $\sigma_{0}$ is an area form on $S^{2}$ with total area 1 , and

$$
M_{\lambda}^{1}=\left(\mathbb{C} P^{2} \# \overline{\mathbb{C} P}^{2}, \omega_{\lambda}^{1}\right), \quad-1<\lambda \in \mathbb{R}
$$

where $\omega_{\lambda}^{1}$ takes the value $2+\lambda$ on the class of the line and $1+\lambda$ on the exceptional divisor. More explicitly, $\omega_{\lambda}^{1}$ can be obtained by collapsing the bounding spheres of the annulus

$$
\left\{z \in \mathbb{C}^{2}: \lambda+1 \leq \pi\left|z^{2}\right| \leq \lambda+2\right\}
$$

along their null foliations by Hopf circles. We will denote the corresponding groups of symplectomorphisms by $G_{\lambda}^{i}, i=0,1$.

As was first observed by Gromov [7, the topological type of $G_{\lambda}^{i}$ changes as $\lambda$ increases. He showed that the identity component of the group

$$
G_{0}^{0}=\operatorname{Symp}\left(S^{2} \times S^{2}, \sigma_{0} \times \sigma_{0}\right)
$$

deformation retracts to the Lie group $\mathrm{SO}(3) \times \mathrm{SO}(3)$, and that the full group is an extension of this Lie group by $\{ \pm 1\}$, where -1 acts by permuting the factors. He also pointed out that as soon as $\lambda$ gets bigger than zero, $G_{\lambda}^{0}$ becomes connected and a new element of infinite order appears in its fundamental group. The key idea in his proof was to look at the action of $G_{\lambda}^{0}$ on the contractible space $\mathcal{J}_{\lambda}^{\prime}$ of $\omega_{\lambda}^{0}$-compatible almost complex structures.

By exploiting this idea further, the first author [1] calculated the rational cohomology ring of $G_{\lambda}^{0}$ for $\lambda$ in the range $0<\lambda \leq 1$. The first main result of the present paper is the completion of this calculation for arbitrary $\lambda$. Recall that the rational cohomology ring $H^{*}(G)$ of a topological group $G$ is a free graded ring. We denote by $\Lambda\left(x_{1}, \ldots, x_{\ell}\right)$ the exterior algebra over $\mathbb{Q}$ on generators $x_{j}$ of odd degree and by $S\left(w_{1}, \ldots, w_{\ell}\right)$ the polynomial algebra on even generators $w_{j}$.

Theorem 1.1. When $\lambda>0$ the group $G_{\lambda}^{0}$ is path-connected, and has fundamental group $\mathbb{Z} \oplus \mathbb{Z} / 2 \mathbb{Z} \oplus \mathbb{Z} / 2 \mathbb{Z}$. Moreover if $0 \leq \ell-1<\lambda \leq \ell$ for some $\ell \in \mathbb{N}$,

$$
H^{*}\left(G_{\lambda}^{0} ; \mathbb{Q}\right)=\Lambda(a, x, y) \otimes S\left(w_{\ell}\right),
$$

where $\operatorname{deg} a=1, \operatorname{deg} x=\operatorname{deg} y=3$ and $\operatorname{deg} w_{\ell}=4 \ell$.

Thus the cohomology remains stable as $\lambda$ varies within an interval of the form $(\ell-1, \ell]$ but jumps as $\lambda$ moves past the endpoint $\ell$. The proof is simply a more elaborate version of the calculation in 11. It is based on the fact that the space $\mathcal{J}_{\lambda}^{0}$ has a natural stratification by sets $U_{j}^{0}$, each of which is homotopy equivalent to a homogeneous space of the group $G_{\lambda}^{0}$. More precisely, the stratum $U_{k}^{0}$ contains the Hirzebruch integrable complex structure $J_{2 k}$ with a holomorphic sphere of selfintersection $-2 k$, and it is (weakly) homotopic to the quotient $G_{\lambda}^{0} / \operatorname{Aut}\left(J_{2 k}\right)$, where $\operatorname{Aut}\left(J_{2 k}\right)$ denotes the stabilizer of $J_{2 k}$; see $\S 2$. This gives rise to a family of MayerVietoris sequences which can be completely analyzed; see Proposition 3.15.

It is easiest to understand what is happening if we consider homotopy, rather than cohomology. We define $K_{k}^{0}$ to be the identity component of $\operatorname{Aut}\left(J_{2 k}\right)$. Thus $K_{0}^{0}$ is the subgroup $\mathrm{SO}(3) \times \mathrm{SO}(3)$ mentioned above, while, for $k>0$, we will see in $\S 2$ that $K_{k}^{0}$ is isomorphic to $S^{1} \times \mathrm{SO}(3)$. In this setting, the generators $\xi, \eta \in \pi_{3}\left(G_{\lambda}\right)$ dual to $x, y$ come from the inclusion of $K_{0}^{0}$ into $G_{\lambda}^{0}$. As $\lambda$ increases above 0 a new 1-dimensional generator $\alpha$ appears that lies in $\pi_{1}\left(K_{1}^{0}\right)$. It does not commute with $\xi$, and so a new auxiliary class $\zeta_{1}$ also appears that is represented 
in homotopy by the Samelson product $[\alpha, \xi]^{1}$ As $\lambda$ increases past 1 , one can find representatives of $\alpha$ and $\xi$ that both lie in $K_{2}^{0}$. Since they commute, the Samelson product $[\alpha, \xi]$ vanishes. Hence there is a 5-disk that bounds $\zeta_{1}$, and the new 8dimensional generator $\zeta_{2}$ is a higher product made from this new disk and $\xi$. It is shown in [3] that such higher products in the group $G$ are the desuspension of higher Whitehead products in $B G$ and hence give rise to relations in the rational cohomology ring of the classifying space $B G$. By pursuing this argument, we prove the following result in $₫[6$

Theorem 1.2. If $0 \leq \ell-1<\lambda \leq \ell$ for some $\ell \in \mathbb{N}$, then

$$
H^{*}\left(B G_{\lambda}^{0} ; \mathbb{Q}\right)=S(A, X, Y) /\left\{A(X-Y)(4 X-Y) \cdots\left(\ell^{2} X-Y\right)=0\right\},
$$

where $\operatorname{deg} A=2$ and $\operatorname{deg} X=\operatorname{deg} Y=4$.

A phenomenon similar to the existence of these "jumping" generators $w_{\ell}$ and $\zeta_{\ell}$ was also discovered by Kronheimer [9] in the guise of some nontrivial families of symplectic forms, detected using properties of Seiberg-Witten invariants. The relation of our work to his is explained in $\$$ [5 Kronheimer's paper was in turn motivated by work of Seidel [17] showing that "many" symplectic 4-manifolds admit symplectic diffeomorphisms which are differentiably isotopic to the identity but not symplectically isotopic. Of course, Theorems 1.1 and 1.4 show that this type of phenomenon does not happen on $S^{2} \times S^{2}$ or $\mathbb{C} P^{2} \# \overline{\mathbb{C P}}^{2}$.

Another interesting question concerns the behavior of the groups $G_{\lambda}^{0}$ as $\lambda \rightarrow \infty$. Since there is no obvious map $G_{\lambda}^{0} \rightarrow G_{\mu}^{0}$ when $\lambda<\mu$ it is not quite clear how to interpret this limit. In $\oiint_{4}$ we show that it can be defined as a bundle of groups

$$
G_{\infty}^{0}=\bigcup_{\lambda>0}\left\{(\phi, \lambda): \phi \in G_{\lambda}^{0}\right\} \subset \operatorname{Diff}\left(S^{2} \times S^{2}\right) \times \mathbb{R}^{+} .
$$

One can think of $G_{\infty}^{0}$ as a topological category (or groupoid) with $\mathbb{R}^{+}$as space of objects and where the set of morphisms from $\lambda$ to $\mu$ is empty unless $\lambda=\mu$, in which case it equals $G_{\lambda}^{0}$. We prove the following result.

Proposition 1.3. $G_{\infty}^{0}$ is homotopy equivalent to the group

$$
\mathrm{SO}(3) \times \operatorname{Map}\left(S^{2}, \mathrm{SO}(3)\right)
$$

where Map denotes the space of unbased smooth maps.

Thus $G_{\infty}^{0}$ is homotopy equivalent to the group $\mathcal{D}$ of all diffeomorphisms of $S^{2} \times S^{2}$ that commute with the projection $p r$ to the first (i.e. the larger) factor. In other words, in the limit the only remaining structure is that of the fibration $p r: S^{2} \times$ $S^{2} \rightarrow S^{2}$. Note that in this limit we allowed the size of the larger sphere to increase without bound, while the smaller sphere has area fixed at 1 . Sometimes it is convenient to reparametrize this, fixing the area of the larger sphere at 1 and allowing the size of the smaller sphere to go to zero. (This is the kind of adiabatic limit considered by Salamon in [16 for example.) In this case it is natural to interpret $\mathcal{D}$ as the group of all diffeomorphisms of $S^{2} \times S^{2}$ that preserve the limiting (degenerate) form $p r^{*}(\sigma)$. Since the group of symplectomorphisms does not change when the symplectic form is multiplied by a constant, these different ways of taking

${ }^{1}$ This is given by the map $S^{4}=S^{1} \times S^{3} / S^{1} \vee S^{3} \rightarrow G_{\lambda}$ induced by the commutator

$$
S^{1} \times S^{3} \rightarrow G_{\lambda}: \quad(s, u) \mapsto \alpha(s) \xi(u) \alpha(s)^{-1} \xi(u)^{-1} .
$$

It is well known to be the desuspension of the Whitehead product on $\pi_{*}(B G)$; see [18]. 
the limit yield the same result. Observe also that an analogous result holds for the symplectomorphism groups of any ruled surface; see Remark 4.7.

There is a completely analogous story for the groups $G_{\lambda}^{1}$ of symplectomorphisms of $\mathbb{C} P^{2} \# \overline{\mathbb{C} P}^{2}$. In this case Gromov's methods show that $G_{\lambda}^{1}$ deformation retracts onto the unitary group $\mathrm{U}(2)$ when $-1<\lambda \leq 0$, and our arguments again show that its cohomology jumps as $\lambda$ passes each integer. This time, however, there already are generators $a, x$ of degrees 1 and 3 in the cohomology of $\mathrm{U}(2)$ and the new persistent generator $y$ that appears has degree 3, while the "jumping generator" has degree $6,10, \ldots$ and so on.

Theorem 1.4. For all $\lambda>-1$ the group $G_{\lambda}^{1}$ is path-connected, and has fundamental group $\mathbb{Z}$. Moreover if $0 \leq \ell-1<\lambda \leq \ell$ for some $\ell \in \mathbb{N}$,

$$
H^{*}\left(G_{\lambda}^{1} ; \mathbb{Q}\right)=\Lambda(a, x, y) \otimes S\left(w_{\ell}\right),
$$

where $\operatorname{deg} a=1, \operatorname{deg} x=\operatorname{deg} y=3$ and $\operatorname{deg} w_{\ell}=4 \ell+2$.

Theorem 1.5. If $0 \leq \ell-1<\lambda \leq \ell$ for some $\ell \in \mathbb{N}$, then

$$
H^{*}\left(B G_{\lambda}^{1} ; \mathbb{Q}\right)=S(A, X, Y) /\left\{(X-Y)(4 X-Y) \cdots\left((\ell+1)^{2} X-Y\right)=0\right\},
$$

where $\operatorname{deg} A=2$ and $\operatorname{deg} X=\operatorname{deg} Y=4$.

Some of these results can be extended to ruled surfaces over arbitrary bases; see [13].

\section{The stratification of $\mathcal{J}_{\lambda}^{i}$ And the groups $K_{k}^{i}$}

In this section we define the stratification of the space $\mathcal{J}_{\lambda}^{i}$ of $\omega_{\lambda}^{i}$-compatible almost complex structures and establish its basic properties. Each stratum is homotopy equivalent to a homogeneous space $G_{\lambda}^{i} / K_{k}^{i}$, where the subgroups $K_{k}^{i}$ are finite dimensional Lie groups. In $\$ 2.5$ we work out the relations between these groups $K_{k}^{i}$.

2.1. Structure of $J$-holomorphic spheres on $M_{\lambda}^{i}$. We first consider $M_{\lambda}^{0}=$ $\left(S^{2} \times S^{2}, \omega_{\lambda}^{0}\right)$. In this case we always take $\ell$ to be the positive integer such that

$$
\ell-1<\lambda \leq \ell \text {. }
$$

Further, $E_{0}$ denotes the homology class of $S^{2} \times\{p t\}, F$ denotes the fiber class $\{p t\} \times S^{2}$, and we define $E_{2 k}=E_{0}-k F$ for $k=0,1,2, \ldots$ General background information about $J$-holomorphic spheres can be found in [1, 10, 14, 15].

Let $J_{0}=j_{0} \oplus j_{0} \in \mathcal{J}_{\lambda}^{0}$ be the standard split compatible complex structure. For $J_{0}$, the classes $E_{0}$ and $F$ are both represented by 2-parameter families of holomorphic spheres given respectively by $S^{2} \times\{s\}$ and $\{s\} \times S^{2}$, for any $s \in S^{2}$. Moreover, by positivity of intersections no class $E_{2 k}$ with $k>0$ can be represented by a $J_{0}$-holomorphic curve. More generally, we have:

Proposition 2.1. $\quad$ (i) For any $J \in \mathcal{J}_{\lambda}^{0}$, the fiber class $F$ is represented by a 2parameter family of embedded $J$-holomorphic spheres that form the fibers of a fibration $S^{2} \times S^{2} \rightarrow S^{2}$. If $\lambda=0$ the same is true for $E_{0}$.

(ii) For each $k, 0 \leq k \leq \ell$, the class $E_{2 k}$ is represented by a $J$-holomorphic sphere for some $J \in \mathcal{J}_{\lambda}^{0}$.

(iii) Conversely, for each $J \in \mathcal{J}_{\lambda}^{0}$, there is an integer $0 \leq k \leq \ell$ such that the class $E_{2 k}$ has a J-holomorphic representative. When $k>0$ this sphere is unique and no other class $E_{2 j}$ with $0 \leq j, j \neq k$, has such a representative. 
Proof. The first statement is a well-known and standard result in the theory of $J$-holomorphic curves; see e.g. [10]. To prove (ii), first note that if $0 \leq k \leq \ell$, then

$$
\omega_{\lambda}^{0}\left(E_{2 k}\right)=1+\lambda-k>0,
$$

which is a necessary condition for $E_{2 k}=E_{0}-k F$ to have a holomorphic representative for some $J \in \mathcal{J}_{\lambda}^{0}$. We show that it does in $\$ 2.3$ by an explicit construction.

The third statement follows from the compactness theorem for $J$-holomorphic curves. Since $\mathcal{J}_{\lambda}^{0}$ is connected there is a path $J_{t}, t \in[0,1]$, connecting the standard split structure $J_{0}$ to any given $J=J_{1}$. For a generic path, the class $E_{0}$ will be represented for all $J_{t}, t<1$, and will degenerate at $t=1$ into some cusp-curve that has to contain a component in some class $E_{2 k}$. Next recall that the principle of positivity of intersections says that each intersection point of two distinct $J$-holomorphic curves in a 4-manifold contributes positively to their intersection number. This implies that the intersection number $A \cdot B$ of any two different classes $A, B$ with $J$-holomorphic representatives must be $\geq 0$. Since

$$
E_{2 k} \cdot E_{2 j}=\left(E_{0}-k F\right) \cdot\left(E_{0}-j F\right)=-k-j,
$$

the uniqueness and nonexistence statements in (iii) follow immediately.

QED

Consider now $M_{\lambda}^{1}=\left(\mathbb{C} P^{2} \# \overline{\mathbb{C} P}^{2}, \omega_{\lambda}^{1}\right)$, with $-1<\lambda \in \mathbb{R}$, described in $\S 1$ Recall that $E_{1}$ denotes the exceptional divisor, with self-intersection -1 , and $F$ is used again to denote the fiber of the fibration $\mathbb{C} P^{2} \# \overline{\mathbb{C P}}^{2} \rightarrow S^{2}$, having self-intersection 0 . Moreover, the symplectic form $\omega_{\lambda}^{1}$ is such that

$$
\omega_{\lambda}^{1}(F)=1 \text { and } \omega_{\lambda}^{1}\left(E_{1}\right)=\lambda+1 .
$$

In this case we also define the integer $\ell$ by the condition

$$
\ell-1<\lambda \leq \ell \text {. }
$$

We further define $E_{2 k+1}$ by $E_{2 k+1}=E_{1}-k F$ for $k=0,1,2, \ldots$ Thus, again, the self-intersection number of $E_{2 k+1}$ is $2 k+1$.

Let $J_{1} \in \mathcal{J}_{\lambda}^{1}$ be the standard compatible complex structure on $\mathbb{C} P^{2} \# \overline{\mathbb{C P}}^{2}$. For $J_{1}$, the class $F$ is represented by a 2-parameter family of holomorphic spheres given by the fibers of the holomorphic fibration $\mathbb{C} P^{2} \# \overline{\mathbb{C} P}^{2} \rightarrow S^{2}$, and the class $E_{1}$ is represented by a unique holomorphic sphere given by the exceptional divisor. Here is the analog of Proposition 2.1. The proof is similar and is left to the reader.

Proposition 2.2. $\quad$ (i) For any $J \in \mathcal{J}_{\lambda}^{1}$, the fiber $F$ is represented by a 2-parameter family of embedded $J$-holomorphic spheres that fiber $\mathbb{C} P^{2} \# \overline{\mathbb{C P}}^{2}$. If $\ell=0, E_{1}$ is represented by a unique embedded $J$-holomorphic sphere for any $J \in \mathcal{J}_{\lambda}^{1}$.

(ii) For each $k, 0 \leq k \leq \ell$, the class $E_{2 k+1}$ is represented by a J-holomorphic sphere for some $J \in \mathcal{J}_{\lambda}^{1}$.

(iii) Conversely, for each $J \in \mathcal{J}_{\lambda}^{1}$, there is an integer $k \leq \ell$ such that the class $E_{2 k+1}$ has a J-holomorphic representative. This sphere is unique and no other class $E_{2 j+1}$ with $0 \leq j, j \neq k$, has such a representative.

2.2. The strata of $\mathcal{J}_{\lambda}^{i}$. As always we fix $\ell \in \mathbb{N}$ and assume that $\ell-1<\lambda \leq \ell$. For each $0 \leq k \leq \ell$ and $i=0,1$, define $U_{k}^{i} \subset \mathcal{J}_{\lambda}^{i}$ as the set of all $\omega_{\lambda}^{i}$-compatible almost complex structures for which the class $E_{i}-k F=E_{2 k+i}$ is represented by a pseudo-holomorphic sphere. A consequence of Propositions 2.1 and 2.2 is that the $U_{k}^{i}$ 's are disjoint subsets of $\mathcal{J}_{\lambda}^{i}$ for $i=0,1$. Throughout we work with $C^{\infty}$-maps and almost complex structures, and so by manifold we mean a Fréchet manifold. 
Proposition 2.3. Let $i=0$ or 1 . Then $U_{0}^{i}$ is an open dense subset of $\mathcal{J}_{\lambda}^{i}$, and each $U_{k}^{i}, 1 \leq k \leq \ell$, is a nonempty submanifold of $\mathcal{J}_{\lambda}^{i}$ of codimension $4 k-2$ if $i=0$ and $4 k$ if $i=1$. Its closure is given by

$$
\bar{U}_{k}^{i}=U_{k}^{i} \cup U_{k+1}^{i} \cup \cdots \cup U_{\ell}^{i} .
$$

In particular,

$$
\mathcal{J}_{\lambda}^{i}=\bar{U}_{0}^{i}=U_{0}^{i} \cup U_{1}^{i} \cup \cdots \cup U_{\ell}^{i} .
$$

Proof. This is a relatively easy result whose proof is analogous to the proof of Theorem 1.8 in [1]. See also $\S 4.1$ in [12].

QED

For the algebraic computations in 43 , we need more information regarding the way the $U_{k}^{i}$, s fit together to give the contractible space $\mathcal{J}_{\lambda}^{i}$. Define $U_{01 \ldots k}^{i}$ for $0 \leq k \leq \ell$ by

$$
U_{01 \ldots k}^{i}=U_{0}^{i} \cup U_{1}^{i} \cup \cdots \cup U_{k}^{i} .
$$

The next result says that these spaces $U$ are stratified. By this we mean that $U$ is a union of a finite number of disjoint manifolds that are called strata. Each stratum $\mathcal{S}$ has a neighborhood $\mathcal{N}_{\mathcal{S}}$ that projects to $\mathcal{S}$ by a map $\mathcal{N}_{\mathcal{S}} \rightarrow \mathcal{S}$. When $\mathcal{N}_{\mathcal{S}}$ is given the induced stratification, this map is a locally trivial fiber bundle whose fiber has the form of a cone $C(\mathcal{L})$ over a finite dimensional stratified space $\mathcal{L}$ that is called the link of $\mathcal{S}$ in $U$. Moreover, $\mathcal{S}$ sits inside $\mathcal{N}_{\mathcal{S}}$ as the set of vertices of all these cones.

Proposition 2.4. $\quad$ (i) For each $0 \leq k \leq \ell$, the space $U_{01 \ldots k}^{i}$ is stratified with strata $U_{j}^{i}, 0 \leq j \leq k$.

(ii) The link of $U_{k}^{i}$ in $U_{01 \ldots k}^{i}$ is the sphere $S^{4 k-3}$ when $i=0$ and $S^{4 k-1}$ when $i=1$. Moreover, if $k>1$ the link $L_{k}^{i}$ of $U_{k}^{i}$ in $U_{k-1}^{i}$ is a smooth 3-manifold (in fact a lens space).

Proof. The first statement is the main result of [12] and is proved by showing that for all $J \in \mathcal{J}_{\lambda}^{i}$ the space of all $J$-holomorphic stable maps into $M_{\lambda}^{i}$ has a corresponding stratification. The first statement in (ii) follows from the previous proposition, while the second can be proved by looking at the gluing parameters necessary to glue in one fiber; see [12]. We give a rough idea of its proof since this will be needed later.

Each element $J \in U_{k}$ defines a fibration $\pi_{J}: M_{\lambda}^{i} \rightarrow S^{2}$ together with a unique $J$-holomorphic section $C_{J}$ in the class $E_{2 k+i}=E_{i}-k F$. There is a corresponding family $\tau_{q}, q \in S^{2}$, of $J$-holomorphic cusp-curves (or stable maps) in class $E_{i}-$ $(k-1) F$. Namely, $\tau_{q}$ is the union of $C_{J}$ with the fiber $F_{q}=\pi_{J}^{-1}(q)$. The gluing process allows one to build a family of $J_{(q, a)}$-holomorphic spheres $S_{(q, a)}$ from the pair of components in $\tau_{q}$, one for each sufficiently small "gluing parameter" $a \in \mathbb{C}_{q}$. Here $\mathbb{C}_{q}$ can be identified with the tensor product of the tangent spaces to the two components $C_{J}$ and $F_{x}$ of $\tau_{q}$ at their intersection point, and so fit together to form a complex line bundle $L$ over $S^{2}$ with Chern class $2-2 k$. The permitted gluing parameters $(q, a)$ fill out a neighborhood $\mathcal{V}$ of its zero section.

Now observe that because $S_{(q, a)}$ lies in the class $E_{i}-(k-1) F$, the corresponding almost complex structures $J_{(q, a)}$ lie in $U_{k-1}$. Further, except in the case $i=0, k=1$, there is a unique $J_{(q, a)}$-holomorphic curve in the class $E_{i}-(k-1) F$, and one can 
show that the map

$$
\mathcal{V} \rightarrow U_{k-1}: \quad(q, a) \mapsto J_{(q, a)}
$$

is a homeomorphism onto a normal slice to $U_{k}$ in $U_{k-1}$ at $J$. Thus the $\operatorname{link} L_{k}^{i}$ is homeomorphic to $\partial \mathcal{V}$ which is the unit sphere bundle of $L$ and so is a lens space.

QED

2.3. The manifolds $M_{\lambda}^{i}$ as symplectic reductions of $\mathbb{C}^{4}$. In order to be able to identify the strata $U_{k}^{i}$ of $\mathcal{J}_{\lambda}^{i}$ with quotients of $G_{\lambda}^{i}$ by appropriate compact subgroups, we now show how to describe $M_{\lambda}^{i}$ as a symplectic reduction of $\mathbb{C}^{4}$. A good reference for this subsection is the article by M. Audin in [6]. For general expositions on symplectic group actions and reduction see, for example, [5] and [14.

Consider the unitary action of $\mathbb{T}^{2}$ on $\mathbb{C}^{4}$ given by

$$
(s, t) \cdot\left(z_{1}, z_{2}, z_{3}, z_{4}\right)=\left(s^{m} t z_{1}, t z_{2}, s z_{3}, s z_{4}\right),|s|=|t|=1,
$$

and let $\phi: \mathbb{C}^{4} \rightarrow \mathbb{R}^{2}$ be the corresponding moment map:

$$
\phi\left(z_{1}, z_{2}, z_{3}, z_{4}\right)=\frac{1}{2}\left(m\left|z_{1}\right|^{2}+\left|z_{3}\right|^{2}+\left|z_{4}\right|^{2},\left|z_{1}\right|^{2}+\left|z_{2}\right|^{2}\right) .
$$

If $\mu>m$ one checks easily that $\xi=(\mu, 1) \in \mathbb{R}^{2}$ is a regular value of $\phi$ and so we can consider the symplectic reduction $\phi^{-1}(\mu, 1) / \mathbb{T}^{2}$ which we denote by $R_{\mu}^{m}$.

Since the reduction is performed with respect to a unitary action of $\mathbb{T}^{2}$, the complex structure of $\mathbb{C}^{4}$ descends naturally to the quotient, giving $R_{\mu}^{m}$ the structure of a Kähler manifold. By looking at the action of $\mathbb{T}^{2}$ on the level set $\phi^{-1}(\mu, 1)$, it is not hard to see that $R_{\mu}^{m}$ can be identified with the space

$$
W_{m}=\left\{\left(\left[u_{0}, u_{1}\right],\left[w_{0}: w_{1}: w_{2}\right]\right): u_{0}^{m} w_{1}=u_{1}^{m} w_{0}\right\} \subset \mathbb{C} P^{1} \times \mathbb{C} P^{2}
$$

via the map

$$
\mathbb{C}^{4} \rightarrow W_{m}:\left(z_{1}, z_{2}, z_{3}, z_{4}\right) \mapsto\left(\left[z_{3}: z_{4}\right],\left[z_{3}^{m} z_{2}: z_{4}^{m} z_{2}: z_{1}\right]\right) .
$$

(See also [6], page 61.) Hence $W_{m}$ fibers over the copy of $S^{2}$ with coordinates $z_{3}, z_{4}$, with fiber the $z_{1}, z_{2}$-sphere. Standard arguments show that when $m$ is even this fiber bundle is trivial, and that when $m$ is odd it is nontrivial.

Next observe that the submanifolds $\widetilde{E}_{m} \subset R_{\mu}^{m}$ defined by

$$
\widetilde{E}_{m}=\left\{\text { reduction of }\left\{z_{2}=0\right\} \subset \mathbb{C}^{4}\right\}
$$

are holomorphic spheres with symplectic area given by $\mu-m$. Thus, when $m=2 k$ is even, $R_{\mu}^{2 k}$ is diffeomorphic to $S^{2} \times S^{2}$ and the submanifold $\widetilde{E}_{2 k}$ represents the class $E_{2 k}=E_{0}-k F$ defined in 2.1. Moreover, if $\mu=1+\lambda+k>2 k$ we have that $R_{1+\lambda+k}^{2 k}$ is symplectomorphic to $M_{\lambda}^{0}$ and, if we define $J_{2 k}$ as the push-forward of the complex structure of $R_{1+\lambda+k}^{2 k}$ under this symplectomorphism, the class $E_{2 k}$ has a $J_{2 k}$-holomorphic representative given by the image of $\widetilde{E}_{2 k}$, i.e. $J_{2 k} \in U_{k}^{0}$. Note that $J_{0}$ defined in this way is the standard split complex structure $j_{0} \oplus j_{0}$ considered in $\$ 2.1$

Similarly, when $m=2 k+1$ is odd, $R_{\mu}^{2 k+1}$ is diffeomorphic to $\mathbb{C} P^{2} \# \overline{\mathbb{C P}}^{2}$ and the submanifold $\widetilde{E}_{2 k+1}$ represents the class $E_{2 k+1}=E_{1}-k F$ also defined in 2.1 . If $\mu=2+\lambda+k>2 k+1$ we also have that $R_{2+\lambda+k}^{2 k+1}$ is symplectomorphic to $M_{\lambda}^{1}$ and, if we define $J_{2 k+1}$ as the push-forward of the complex structure of $R_{2+\lambda+k}^{2 k+1}$ under 
this symplectomorphism, the class $E_{2 k+1}$ has a $J_{2 k+1}$-holomorphic representative given by the image of $\widetilde{E}_{2 k+1}$, i.e. $J_{2 k+1} \in U_{k}^{1}$.

We now want to understand the isometries of the above Kähler structures on $M_{\lambda}^{0}$ and $M_{\lambda}^{1}$. When $m=0$, the subgroup $\mathrm{SU}(2) \times \mathrm{SU}(2) \subset \mathrm{U}(4)$ given by matrices of the form

$$
\left(\begin{array}{cc}
A & 0 \\
0 & D
\end{array}\right) \text { with } A, D \in S U(2) \text {, }
$$

acts on $\mathbb{C}^{4}$ by matrix multiplication, preserving the moment map $\phi$ and commuting with the $\mathbb{T}^{2}$ action defined above. Since the intersection of these two subgroups of $\mathrm{U}(4)$ is $\{ \pm 1\} \times\{ \pm 1\}$, we get an effective Kähler action of $\mathrm{SO}(3) \times \mathrm{SO}(3) \cong$ $\mathrm{SU}(2) /\{ \pm 1\} \times \mathrm{SU}(2) /\{ \pm 1\}$ on $R_{\mu}^{0}$, which is nothing else than the standard action of $\mathrm{SO}(3) \times \mathrm{SO}(3)$ on $S^{2} \times S^{2}$ (see [8]).

When $m>0$, the above construction can still be done if we restrict ourselves to the subgroup $\mathrm{SU}(2) \times S^{1} \subset \mathrm{U}(4)$ given by matrices of the form

$$
\left(\begin{array}{cc}
A & 0 \\
0 & D
\end{array}\right) \text { with } A=\left(\begin{array}{cc}
\alpha & 0 \\
0 & \bar{\alpha}
\end{array}\right), \quad \alpha \bar{\alpha}=1, \text { and } D \in \mathrm{SU}(2) .
$$

This subgroup also acts on $\mathbb{C}^{4}$ by matrix multiplication, preserving the moment map $\phi$ and commuting with the above $\mathbb{T}^{2}$ action.

When $m=2 k$ is even, the intersection of these two subgroups of $\mathrm{U}(4)$ is again $\{ \pm 1\} \times\{ \pm 1\}$ and we get an effective Kähler action of $\mathrm{SO}(3) \times S^{1} \cong \mathrm{SU}(2) /\{ \pm 1\} \times$ $S^{1} /\{ \pm 1\}$ on $R_{\mu}^{2 k}$. Moreover, the submanifold $\tilde{E}_{2 k} \subset R_{\mu}^{2 k}$ is a connected component of the fixed point set of the $S^{1}$ part of this action. Taking $\mu=1+\lambda+k$ and using the fact that $R_{1+\lambda+k}^{2 k}$ is symplectomorphic to $M_{\lambda}^{0}$, we have constructed this way an $\mathrm{SO}(3) \times S^{1}$ Kähler action on $M_{\lambda}^{0}$, with complex structure $J_{2 k} \in U_{k}^{0}$, such that each point of the $J_{2 k}$-holomorphic representative for the class $E_{2 k}$ is fixed by the $S^{1}$ part of the action. Note that when $k=1$ the $\mathrm{SO}(3)$ part of this action is the same as the diagonal $\mathrm{SO}(3)$ action on $S^{2} \times S^{2}$ (see [1).

When $m=2 k+1$ is odd, the intersection of the above $\mathrm{SU}(2) \times S^{1} \subset \mathrm{U}(4)$ and $\mathbb{T}^{2} \subset \mathrm{U}(4)$ is given by $\{(1, \pm 1),(-1, \pm i)\}$, and so we get an effective Kähler action of $\mathrm{U}(2) \cong \mathrm{SU}(2) \times S^{1} /\{(1, \pm 1),(-1, \pm i)\}$ on $R_{\mu}^{2 k+1}$. Moreover, the submanifold $\tilde{E}_{2 k+1} \subset R_{\mu}^{2 k+1}$ is a connected component of the fixed point set of the natural $S^{1}$-subgroup that this description of $\mathrm{U}(2)$ gives:

$$
S^{1} \cong S^{1} /\{ \pm 1\} \hookrightarrow\{1\} \times S^{1} /\{ \pm 1\} \hookrightarrow \mathrm{SU}(2) \times S^{1} /\{(1, \pm 1),(-1, \pm i)\} .
$$

Taking $\mu=2+\lambda+k$ and using the fact that $R_{2+\lambda+k}^{2 k+1}$ is symplectomorphic to $M_{\lambda}^{1}$, we have constructed this way a $\mathrm{U}(2)$ Kähler action on $M_{\lambda}^{1}$, with complex structure $J_{2 k+1} \in U_{k}^{1}$, such that the $J_{2 k+1}$-holomorphic representative for the class $E_{2 k+1}$ is fixed by a given $S^{1}$ subgroup of $\mathrm{U}(2)$.

We have thus proved the following:

Proposition 2.5. (i) For each $0<k \leq \ell, M_{\lambda}^{0}$ has a symplectic $K_{k}^{0} \cong \mathrm{SO}(3) \times S^{1}$ action, which is Kähler for a standard complex structure $J_{2 k} \in U_{k}^{0}$ and is such that the $J_{2 k}$-holomorphic representative $C_{2 k}$ for the class $E_{2 k}$ is fixed by the $S^{1}$ part of the action.

(ii) For each $0 \leq k \leq \ell, M_{\lambda}^{1}$ has a symplectic $K_{k}^{1} \cong \mathrm{U}(2)$ action, which is Kähler for a standard complex structure $J_{2 k+1} \in U_{k}^{1}$ and such that the $J_{2 k+1}$ holomorphic representative $C_{2 k+1}$ for the class $E_{2 k+1}$ is fixed by a given $S^{1}$ subgroup of $\mathrm{U}(2)$. 
2.4. Geometric description of the strata. The next task is to identify the stratum $U_{k}^{i}$ of $\mathcal{J}_{\lambda}^{i}$ with the quotient of $G_{\lambda}^{i}$ by the isometry group $K_{k}^{i}$.

Proposition 2.6. (i) If $\lambda>0$, the stratum $U_{0}^{0} \subset \mathcal{J}_{\lambda}^{0}$ is weakly homotopy equivalent to $G_{\lambda}^{0} / \mathrm{SO}(3) \times \mathrm{SO}(3)$.

(ii) Each stratum $U_{k}^{0}$ of $\mathcal{J}_{\lambda}^{0}, 1 \leq k \leq \ell$, is weakly homotopy equivalent to $G_{\lambda}^{0} / K_{k}^{0}$, where $K_{k}^{0} \cong \mathrm{SO}(3) \times S^{1}$ is the group of Kähler isometries of a standard complex structure $J_{2 k} \in U_{k}^{0}$.

(iii) Each stratum $U_{k}^{1}$ of $\mathcal{J}_{\lambda}^{1}, 0 \leq k \leq \ell$, is weakly homotopy equivalent to $G_{\lambda}^{1} / K_{k}^{1}$, where $K_{k}^{1} \cong \mathrm{U}(2)$ is the group of Kähler isometries of a standard complex structure $J_{2 k+1} \in U_{k}^{1}$.

Proof. Part (i) was proved in [1]. When $\lambda=0$ the corresponding statement was established by Gromov in 7 . Here the group $K_{0}^{0}=\mathrm{SO}(3) \times \mathrm{SO}(3)$ is not the full isometry group of $J_{0}$ : one has to add in the involution that interchanges the two sphere factors. Gromov then showed that the quotient of $G_{0}^{0}$ by this isometry group is $U_{0}^{0}$, which in this case is contractible since it is the whole of $\mathcal{J}_{0}^{0}$.

The case $k=1$ of part (ii) was proved in [1. Since that proof generalizes directly to any $k$, with $1 \leq k \leq \ell$, we just recall its main steps adapted to this more general context.

One denotes by $\mathcal{S}_{k}^{0}$ the space of embedded symplectic 2-spheres in $M_{\lambda}^{0}$ representing the homology class $E_{2 k}$. Associated to any $J \in U_{k}^{0}$ we have a well-defined element of $\mathcal{S}_{k}^{0}$ given by the unique $J$-sphere representing $E_{2 k}$. Conversely, given any element in $\mathcal{S}_{k}^{0}$ the space of all $J \in U_{k}^{0}$ that make it $J$-holomorphic is nonempty and contractible. It follows that $U_{k}^{0}$ is weakly homotopy equivalent to $\mathcal{S}_{k}^{0}$.

The next step is to prove that $G_{\lambda}^{0}$ acts transitively on $\mathcal{S}_{k}^{0}$. Here is an outline of the method. First of all, given two curves $C_{j}, j=1,2$, in $\mathcal{S}_{k}^{0}$, choose elements $J_{j}$ in $\mathcal{J}_{\lambda}^{0}$ so that $C_{j}$ is $J_{j}$-holomorphic. Then $C_{j}$ is a section of the fibration of $M_{\lambda}^{0}$ formed by the $J_{j}$-holomorphic spheres in class $F$. It follows that there is a diffeomorphism $\phi$ of $M_{\lambda}^{0}$ that takes $C_{1}$ to $C_{2}$. It is easy to adjust $\phi$ so that it is a symplectomorphism near $C_{1}$, and it can be adjusted to be a symplectomorphism everywhere because any two symplectic forms on the complement $M_{\lambda}^{0}-C_{1}$ that are standard near infinity are symplectomorphic. The last step can be justified by explicit geometric arguments as in [1] or by following the ideas in [1].

Denoting by $H_{k}^{0}$ the subgroup of $G_{\lambda}^{0}$ consisting of symplectomorphisms that preserve the unique $J_{2 k}$-holomorphic sphere in the class $E_{2 k}$, we then have that $G_{\lambda}^{0} / H_{\lambda}^{0} \cong \mathcal{S}_{k}^{0}$. The obvious map

$$
G_{\lambda}^{0} / K_{k}^{0} \rightarrow G_{\lambda}^{0} / H_{k}^{0}
$$

is a fibration, with fiber over the identity given by $H_{k}^{0} / K_{k}^{0}$. The last step of the proof of (ii) is to show that $H_{k}^{0} / K_{k}^{0}$ is weakly contractible. As in the proof of Lemma 3.6 in [1] for the case $k=1$, one proves first that $H_{k}^{0} / K_{k}^{0}$ is homotopy equivalent to the subgroup of $G_{\lambda}^{0}$ consisting of symplectomorphisms that restrict to the identity on a neighborhood of the sphere $C_{2 k}$, and then shows that this last subgroup is weakly contractible.

Part (iii) of the theorem follows from the same steps as part (ii).

QED

We are now in a position to calculate $\pi_{0}$ and $\pi_{1}$ of the groups $G_{\lambda}^{i}$. Since we already know the homotopy types of $G_{0}^{0}$ and $G_{\lambda}^{1},-1<\lambda \leq 0$ (namely, an extension of $\mathrm{SO}(3) \times \mathrm{SO}(3)$ and $\mathrm{U}(2))$, we will concentrate on the other cases. 
Corollary 2.7. (i) The group $G_{\lambda}^{i}$ is connected, except when $i=\lambda=0$.

(ii) When $\lambda>0, \pi_{1}\left(G_{\lambda}^{0}\right)=\mathbb{Z} \oplus \mathbb{Z} / 2 \mathbb{Z} \oplus \mathbb{Z} / 2 \mathbb{Z}$.

(iii) When $\lambda>0, \pi_{1}\left(G_{\lambda}^{1}\right)=\mathbb{Z}$.

Proof. The previous proposition implies that for $\lambda>0$ the sequence

$$
K_{k}^{i} \rightarrow G_{\lambda}^{i} \rightarrow U_{k}^{i}
$$

is a homotopy fibration, in the sense that there is a corresponding long exact sequence of homotopy groups. Further $\pi_{0}\left(K_{0}^{i}\right)=0$.

Since the strata $U_{k}^{i}, k>0$, have codimension at least 2 in $\mathcal{J}_{\lambda}^{i}$, the top stratum $U_{0}^{i}$ is always path connected. Hence $\pi_{0}\left(G_{\lambda}^{i}\right)=\pi_{0}\left(K_{0}^{i}\right)=0$. Similarly, when $i=1$, the fact that the strata $U_{k}^{1}, k>0$, have codimension at least 4 in $\mathcal{J}_{\lambda}^{1}$ implies that $U_{0}^{1}$ is 2-connected. Hence

$$
\pi_{1}\left(G_{\lambda}^{1}\right)=\pi_{1}\left(K_{0}^{1}\right)=\pi_{1}(\mathrm{U}(2))=\mathbb{Z} .
$$

However, the stratum $U_{1}^{0}$ has codimension 2 in $\mathcal{J}_{\lambda}^{0}$ which means that there is a potential generator of $\pi_{1}\left(U_{0}^{0}\right)$ given by the loop which circles once around $U_{1}^{0}$. The argument in [1] which shows that this loop generates $\pi_{1}\left(U_{0}^{0}\right)$ when $\lambda \leq 1$ goes through in the case $\lambda>1$ without change. The rest of (ii) also follows as in [1].

QED

Corollary 2.8. Each stratum $U_{k}^{i}$ is connected.

Proof. This follows from Proposition 2.6 and the fact that the groups $G_{\lambda}^{i}$ are connected except when $i=\lambda=0$.

QED

The group $K_{k}^{i}$ is defined to be the identity component of the stabilizer of the element $J_{2 k+i}$ of the stratum $U_{k}^{i}$. We now show that it acts transitively on the link $L_{k}^{i}$ of $U_{k}^{i}$ in $U_{k-1}^{i}$ at the point $J_{2 k+i}$. The case $i=0, k=1$ is special, since here the link is a circle. In all other cases the link has dimension 3 .

Proposition 2.9. When $k+i>1$ the group $K_{k}^{i}$ acts transitively on the link $L_{k}^{i}$ of $U_{k}^{i}$ in $U_{k-1}^{i}$ at $J=J_{2 k+i}$.

Proof. This is immediate from the description of this link given in Proposition 2.4 The group $K_{k}^{i}$ acts transitively on the sphere $C_{J}$ (where $J=J_{2 k+i}$ ) and on its unit tangent bundle. It also acts transitively on the unit tangent bundle to the fibers of $\pi_{J}$ at their point of intersection with $C_{J}$. The bundle $L$ of gluing parameters is the tensor product of these two bundles, and it is not hard to see that the action there is transitive too.

QED

2.5. Relations between the $K_{k}^{i}$. We now discuss the relations between the elements in $\pi_{1}\left(G_{\lambda}^{i}\right)$ and $H_{*}\left(G_{\lambda}^{i}\right)$ represented by the homotopy and homology of the different subgroups $K_{k}^{i}$ in $G_{\lambda}^{i}$, beginning as usual with the case $i=0$. Our arguments use some results from 3.1 and 4 . However, the conclusions will not be used until we calculate $H^{*}\left(B G_{\lambda}\right)$ in $\$$.

It will be convenient to use the fact, proved in $\$ 4$, that the $G_{\lambda}^{0}$ have a limit at infinity $G_{\infty}^{0}$ that is homotopy equivalent to the group $\mathcal{D}=\mathcal{D}^{0}$ of orientation preserving fiberwise diffeomorphisms of $S^{2} \times S^{2}$. Since Diff ${ }^{+}\left(S^{2}\right)$ deformation retracts to $\mathrm{SO}(3), \mathcal{D}$ is homotopy equivalent to the product

$$
\mathcal{D}=\mathrm{SO}(3) \times \operatorname{Map}\left(S^{2}, \mathrm{SO}(3)\right),
$$


where $\operatorname{Map}(Y, Z)$ denotes the space of smooth maps from $Y$ to $Z$. It is easy to see from the explicit description given in $\$ 2.3$ of the action of $K_{k}$ on $W_{2 k}$ that this action commutes with the projection onto the $z_{3}, z_{4}$ coordinates, i.e. with the projection of $S^{2} \times S^{2}$ onto its first factor. Thus each $K_{k}^{0}$ is a subgroup of $\mathcal{D}$.

The group $K_{0}^{0}$ is simply $\mathrm{SO}(3) \times \mathrm{SO}(3)$ and we will denote the two obvious generators of $\pi_{1}\left(K_{0}^{0}\right)$ by $\tau$ and $\tau^{\prime}$. For $k>0$ let $\alpha_{k}$ be the element of $\pi_{1}\left(K_{k}^{0}\right)$ corresponding to the $S^{1}$ action that fixes the sphere $C_{2 k}$. Further, let $\xi=\xi_{0}$ and $\eta$ be the two obvious spherical generators of $H_{3}\left(K_{0}^{0}, \mathbb{Z}\right)$, and let $\xi_{k}$, for $k>0$, be the spherical generator of $H_{3}\left(K_{k}^{0}, \mathbb{Z}\right)$. Thus these classes are represented by suitable images of $\mathrm{SU}(2)=S^{3}$ in the groups $K_{k}^{0}$.

Lemma 2.10. For each $k>0$,

(i) $\alpha_{k}=k \alpha_{1}+(k-1) \tau^{\prime} \in \pi_{1}\left(G_{\lambda}\right)$,

(ii) $\xi_{k}=\xi_{0}+k^{2} \eta \in H_{3}\left(G_{\lambda} ; \mathbb{Q}\right)$.

Proof. (i) We saw in Corollary 2.7 that $\pi_{1}\left(G_{\lambda}^{0}\right)=\mathbb{Z} \oplus \mathbb{Z} / 2 \mathbb{Z} \oplus \mathbb{Z} / 2 \mathbb{Z}$, with generators lying in $\pi_{1}\left(K_{k}^{0}\right)$ for $k=0,1$. We claim that $\pi_{1}(\mathcal{D})$ is also equal to $\mathbb{Z} \oplus \mathbb{Z} / 2 \mathbb{Z} \oplus \mathbb{Z} / 2 \mathbb{Z}$ and has the same generators. To see this, note first that $\tau$ generates the copy of $\mathbb{Z} / 2 \mathbb{Z}$ coming from the base, i.e. from the fundamental group of the $\mathrm{SO}(3)$-factor in $\mathcal{D}$. Elements of $\pi_{1}\left(\operatorname{Map}\left(S^{2}, \mathrm{SO}(3)\right)\right)$ correspond to homotopy classes of based maps

$$
S^{1} \vee S^{3}=S^{1} \times S^{2} /\{0\} \times S^{2} \longrightarrow \mathrm{SO}(3),
$$

i.e. to elements of

$$
\pi_{0}\left(\operatorname{Map}_{*}\left(S^{1} \vee S^{3}, \mathrm{SO}(3)\right)\right)=\mathbb{Z} / 2 \mathbb{Z} \oplus \mathbb{Z},
$$

where $\mathrm{Map}_{*}$ is the based mapping space. This has generators

$$
\tau^{\prime} \in \pi_{0}\left(\operatorname{Map}_{*}\left(S^{1}, \mathrm{SO}(3)\right)\right)=\mathbb{Z} / 2 \mathbb{Z}, \quad \alpha_{0} \in \pi_{0}\left(\operatorname{Map}_{*}\left(S^{3}, \mathrm{SO}(3)\right)\right)=\mathbb{Z} .
$$

Thus $\pi_{1}(\mathcal{D}) \cong \mathbb{Z} \oplus \mathbb{Z} / 2 \mathbb{Z} \oplus \mathbb{Z} / 2 \mathbb{Z}$. The two generators $\tau, \tau^{\prime}$ of order 2 lie in $\pi_{1}\left(K_{0}^{0}\right)$, and it is not hard to check that $\alpha_{0}=\alpha_{1}+\tau^{\prime}$, so that $\alpha_{0} \in \pi_{1}\left(K_{1}^{0}\right)$ as claimed.

It follows that the composite

$$
G_{\lambda}^{0} \longrightarrow G_{\infty}^{0} \stackrel{\simeq}{\longrightarrow} \mathcal{D}
$$

induces an isomorphism on $\pi_{1}$. This means that we can work out the relations between the $\alpha_{k}$ in $\pi_{1}(\mathcal{D})$, i.e. we do not need to insist that all the diffeomorphisms we consider are symplectic. In particular we can alter $C_{2 k}$ by an isotopy so that it has the form

$$
C_{2 k}=\left\{\left(z, \rho_{k}(z)\right): z \in S^{2}\right\},
$$

where $\rho_{k}: S^{2} \rightarrow S^{2}$ is any convenient degree $-k$ map. (This corresponds to conjugating the original representative of $\alpha_{k}$ by an element of $\mathcal{D}$.) Since the loop $\alpha_{k}$ is represented by rotations of the fiber that fix $C_{2 k}$, we can represent $\alpha_{k}$ by the loop

$$
\theta \cdot(z, w)=\left(z, R_{\left(\rho_{k}(z), \theta\right)} w\right), \quad \theta \in S^{1}=\mathbb{R} / \mathbb{Z},
$$

where $R_{(w, \theta)}$ is the rotation by $\theta$ about the axis through $w$. It is easy to see that the corresponding element in $\pi_{0}\left(\operatorname{Map}_{*}\left(S^{1} \vee S^{3}, \mathrm{SO}(3)\right)\right)$ is $\tau^{\prime}+k \alpha_{0}$, since the induced map $S^{3} \rightarrow \mathrm{SO}(3)$ has degree $k$. This implies that $\alpha_{k}=k \alpha_{1}+(k-1) \tau^{\prime}$ as claimed.

To prove (ii), we must first consider the effect of the map $G_{\lambda}^{0} \rightarrow \mathcal{D}$ on $H_{3}$. Now $H_{3}(\mathcal{D})=\mathbb{Q} \oplus \mathbb{Q}$ with generators $\xi_{0}, \eta$ from $H_{3}\left(K_{0}^{0}\right)$. Since $H_{2}\left(U_{0}\right)=H_{3}\left(U_{0}\right)=0$ by Corollary 3.5 Proposition 3.1 implies that $H_{3}\left(G_{\lambda}^{0}\right) \cong H_{3}\left(K_{0}^{0}\right)$. Hence the map 
$G_{\lambda}^{0} \rightarrow \mathcal{D}$ induces an isomorphism on $H_{3}$. Thus, again, we can work out the relations between the $\xi_{k}$ in $\mathcal{D}$.

Since the $\mathrm{SO}(3)$-factor in $K_{k}^{0}$ is a lifting to $S^{2} \times S^{2}$ of the usual action on $S^{2}, \xi_{k}$ is represented by a map of the form

$$
\Psi: \mathrm{SO}(3) \rightarrow G_{\lambda}: \quad \Psi(g) \cdot(z, w)=\left(g(z), h_{k, g, z} w\right), \quad g, h_{k, g, z} \in \mathrm{SO}(3) .
$$

Moreover, because all these diffeomorphisms map the section

$$
C_{2 k}=\left\{\left(z, \rho_{k}(z)\right): z \in S^{2}\right\}
$$

into itself,

$$
h_{k, g, z}\left(\rho_{k}(z)\right)=\rho_{k}(g(z)) .
$$

By our earlier remarks, $\rho_{k}$ can be any map of degree $-k$. In particular we may suppose that it has a fixed point $z_{0}$. Let $e: \mathrm{SO}(3) \rightarrow S^{2}$ be the evaluation map at $z_{0}$ and define $F_{k}$ by

$$
F_{k}: \mathrm{SO}(3) \rightarrow \mathrm{SO}(3): \quad g \mapsto h_{k, g, z_{0}} .
$$

Equation (1) implies that there is a commutative diagram:

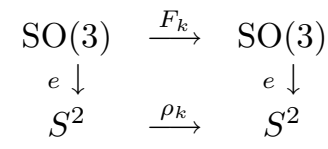

Thus $F_{k}$ preserves the fibers of $e$ and covers a map on the base of degree $-k$. Since it has to commute with the boundary map of the homotopy long exact sequence of $e$, it must also have degree $-k$ on the fiber. Therefore $F_{k}$ has degree $k^{2}$.

Now recall that, for any topological group $G$, the sum of any two spherical cycles $\alpha, \beta$ in $H_{k}(G)$ is represented by the product map

$$
S^{k} \rightarrow G: \quad g \mapsto \alpha(g) \beta(g) .
$$

Hence, since the $\xi_{k}$ are spherical, the class $\xi_{k}-\xi_{0}$ is represented by the map

$$
\mathrm{SO}(3) \rightarrow G_{\lambda}: \quad g \cdot(z, w)=\left(z, h_{k, g, z}(w)\right), h_{k, g, z}, g \in S^{3},
$$

and hence by the map

$$
\mathrm{SO}(3) \rightarrow \operatorname{Map}\left(S^{2}, \mathrm{SO}(3)\right): \quad g \mapsto\left(z \mapsto h_{k, g, z}\right) .
$$

Similarly, $y$ is represented by

$$
\mathrm{SO}(3) \rightarrow \operatorname{Map}\left(S^{2}, \mathrm{SO}(3)\right): \quad g \mapsto(z \mapsto g) .
$$

To check the identity $\xi_{k}-\xi_{0}=k^{2} \eta$ we just have to see that $\xi_{k}-\xi_{0}$ and $k^{2} \eta$ induce the same map on rational $H_{3}$. Since the second loop space $\Omega^{2}(\mathrm{SO}(3))$ has the same rational homotopy type as $S^{1}$, the evaluation map

$$
\operatorname{Map}\left(S^{2}, \mathrm{SO}(3)\right) \rightarrow \mathrm{SO}(3): \quad f \mapsto f\left(z_{0}\right)
$$

induces an isomorphism on $H_{3}(\cdot ; \mathbb{Q})$. Thus the desired conclusion follows from the fact that $F_{k}$ has degree $k^{2}$.

QED

Next let us consider the analogous questions for the case $i=1$. Here it is convenient to identify $\mathbb{C} P^{2} \# \overline{\mathbb{C} P}^{2}$ with the set

$$
\left\{(z, w) \in D^{2} \times S^{2}:\left(e^{i \theta}, w\right) \equiv\left(1, R_{-\theta} w\right), e^{i \theta} \in \partial D^{2}\right\},
$$

where $R_{\theta}$ denotes the rotation of $S^{2}$ about its vertical axis by the angle $\theta \in \mathbb{R} / 2 \pi \mathbb{Z}$, and we identify $D^{2} / \partial D^{2}$ with $S^{2}$ so that the point $\partial D^{2}$ corresponds to the south 
pole $P_{s}$. Then one can check that the section $D^{2} \times\left\{P_{s}\right\}$ has self-intersection -1 so that it can be identified with $C_{1}$.

In this case all groups $K_{k}^{1}$ are isomorphic to $\mathrm{U}(2)$. Further, the group $\mathcal{D}=$ $\mathcal{D}^{1}$ of orientation preserving fiberwise diffeomorphisms of $\mathbb{C} P^{2} \# \overline{\mathbb{C P}}^{2}$ is homotopy equivalent to the semi-direct product

$$
\mathrm{SU}(2) \times_{\mathbb{Z} / 2 \mathbb{Z}} \Gamma\left(S^{2} ; \mathrm{SO}(3)\right),
$$

where the $\mathrm{SU}(2)$ factor is identified with a subgroup of $K_{0}^{1}, \Gamma\left(S^{2} ; \mathrm{SO}(3)\right)$ denotes the space of sections of the bundle over $S^{2}$ with fiber $\mathrm{SO}(3)$ that is associated to the fibration $\mathbb{C} P^{2} \# \overline{\mathbb{C P}}^{2} \rightarrow S^{2}$, and $\mathbb{Z} / 2 \mathbb{Z}$ acts in a way that we now explain. If $R_{\theta}$ denotes the rotation of $S^{2}$ about its vertical axis by the angle $\theta \in \mathbb{R} / 2 \pi \mathbb{Z}$, it is easy to see that

$$
\Gamma\left(S^{2} ; \mathrm{SO}(3)\right)=\left\{\alpha \in \operatorname{Map}\left(D^{2}, \mathrm{SO}(3)\right): \alpha\left(e^{i \theta}\right)=R_{\theta} \alpha(1) R_{-\theta}, e^{i \theta} \in \partial D^{2}\right\} .
$$

With this identification, the generator of $\mathbb{Z} / 2 \mathbb{Z}$ acts by conjugation by $R_{\pi}$ in $\Gamma\left(S^{2} ; \mathrm{SO}(3)\right)$ and by multiplication by $-\mathrm{Id}$ in $\mathrm{SU}(2)$. To see this, compare the present description of $K_{k}$ with that given just before Proposition 2.5. To make the two descriptions more alike, think of $\Gamma\left(S^{2} ; \mathrm{SO}(3)\right)$ as the quotient of $\Gamma\left(S^{2} ; \mathrm{SU}(2)\right)$ by the action of $\mathbb{Z} / 2 \mathbb{Z}$. Then the nontrivial action of $\mathbb{Z} / 2 \mathbb{Z}$ on both factors of $\mathrm{SU}(2) \times \Gamma\left(S^{2} ; \mathrm{SO}(3)\right)$ described above corresponds to the fact that the intersection of $\mathrm{SU}(2) \times S^{1}$ with $\mathbb{T}^{2}$ does not split, even after quotienting out by the $\mathbb{Z} / 2 \mathbb{Z}$ subgroup \pm 1 of $S^{1}$.

Denote by $\alpha_{k}, k \geq 0$, the generator of $\pi_{1}\left(K_{k}^{1}\right)$ corresponding to the $S^{1}$ action that fixes the sphere $C_{2 k+1}$, and by $\xi_{k}$ the generator of $H_{3}\left(K_{k}^{1} ; \mathbb{Z}\right)$ coming from the inclusion of $\mathrm{SU}(2)$ into $\mathrm{U}(2)$. Further, set $\eta=\xi_{1}-\xi_{0}$.

Lemma 2.11. (i) $\alpha_{k}=(k+1) \alpha_{0} \in \pi_{1}\left(G_{\lambda}^{1}\right)$,

(ii) $\xi_{k}=\xi_{0}+k^{2} \eta \in H_{3}\left(G_{\lambda}^{1} ; \mathbb{Z}\right)$.

Proof. The proof of (i) is left to the reader.

Note that there is a fibration

$$
\Omega^{2}(\mathrm{SO}(3)) \longrightarrow \Gamma\left(S^{2} ; \mathrm{SO}(3)\right) \stackrel{e v}{\longrightarrow} \mathrm{SO}(3) .
$$

where $e v(\alpha)=\alpha(1)$. Hence $e v$ induces an isomorphism on $H_{3}$, and so $H_{3}(\mathcal{D})$ has again rank 2. The formula in (ii) now follows as in the previous lemma. Observe that the analog of the set of elements $h_{k, g, z_{0}}, g \in \mathrm{SO}(3)$, is a section of a nontrivial bundle. However, if we take $z_{0}$ to be the point $1 \in \partial D^{2}$ (which corresponds to the south pole $P_{s}$ in $S^{2}$ ) and assume as before that the maps $\rho_{k}$ fix $z_{0}$, then the composites $h_{0, g, g\left(z_{0}\right)}^{-1} \circ h_{k, g, z_{0}}$ are elements of $\mathrm{SU}(2)$ and the argument can proceed as before.

QED

\section{Algebraic computations}

This section is devoted to the algebraic computations necessary to prove the main theorems of this paper. In $\$ 3.1$ we analyse the case of $M_{\lambda}^{0}=\left(S^{2} \times S^{2}, \omega_{\lambda}^{0}\right)$ in complete detail. The case $i=1$ is treated in \$3.2. It is completely analogous to the case $i=0$. 
3.1. Computation of $H^{*}\left(G_{\lambda}^{0}\right)$. As before, we assume $\ell-1<\lambda \leq \ell$. Since this whole subsection is devoted to $M_{\lambda}^{0}=\left(S^{2} \times S^{2}, \omega_{\lambda}^{0}\right)$, we will omit the superscript ${ }^{0}$ to simplify notation. Unless noted otherwise, we assume rational coefficients throughout.

Our strategy is first to show that there is a subalgebra of $H^{*}\left(G_{\lambda}\right)$ isomorphic to $\Lambda(a, x, y) \otimes S\left(w_{\ell}\right)$ and then to show that this subalgebra must be the whole cohomology ring. Thus the first goal is to show how the new element $w_{\ell}$ appears. To do this we use the stratification of the space $\mathcal{J}_{\lambda}$ to construct an interconnected family of modified Mayer-Vietoris sequences $\left(M V_{k}\right)$ relating the cohomology groups of the different strata. This allows us to pinpoint exactly where $w_{\ell}$ comes from; see Definition 3.10 The geometric significance of this generator is discussed further in \$5. In order to show that $\Lambda(a, x, y) \otimes S\left(w_{\ell}\right)$ is the whole of $H^{*}\left(G_{\lambda}\right)$, we calculate all the maps in the part of $\left(M V_{k}\right)$ generated by this subalgebra, and then show that if $d$ is the minimal degree in which $H^{*}\left(G_{\lambda}\right)$ contains something else, there must in fact be a new element in some degree $<d$.

Key to the whole process is the fact that each stratum $U_{k}$ is (weakly homotopic to) a homogeneous space for $G_{\lambda}$. Our first proposition shows that the corresponding decompositions for $H^{*}\left(G_{\lambda}\right)$ are compatible with its multiplicative as well as its additive structure.

Proposition 3.1. $\quad$ (i) $H^{*}\left(G_{\lambda}\right)$ is a free algebra. Moreover, for all $0 \leq k \leq \ell$,

$$
H^{*}\left(G_{\lambda}\right) \cong H^{*}\left(U_{k}\right) \otimes H^{*}\left(K_{k}\right),
$$

as graded algebras.

(ii) For all $k>1, H^{*}\left(U_{k}\right) \cong H^{*}\left(U_{1}\right)$.

Proof. Since $G_{\lambda}$ is a topological group, in particular an $H$-space, we have that $H^{*}\left(G_{\lambda}\right)$ is a Hopf algebra. The Leray Structure Theorem for Hopf algebras over a field of characteristic 0 then says that $H^{*}\left(G_{\lambda}\right)$ is the tensor product of exterior algebras with generators of odd degree and polynomial algebras with generators of even degree (see [18] for relevant definitions and a proof of this structure theorem). Hence $H^{*}\left(G_{\lambda}\right)$ is a free algebra.

The algebra isomorphism (2) was proved in [1 for $k=0$, and the proof for general $k$ is very similar. The first point is that the inclusion $i: K_{k} \rightarrow G_{\lambda}$ is injective in homology. The result for $H_{1}\left(K_{k}\right)$ holds because, as in the proof of Lemma 2.10, the generator $t_{k}$ of $\pi_{1}\left(K_{k}\right) \otimes \mathbb{Q}$ does not vanish in the space of homotopy equivalences of $S^{2} \times S^{2}$. The result for $H_{3}\left(K_{k}\right)$ is somewhat subtle, and uses the fact that $G_{\lambda}$ maps onto the space of homotopy equivalences of each sphere factor by maps of the form:

$$
G_{\lambda} \rightarrow \operatorname{Map}\left(S^{2}, S^{2}\right):\left.\quad g \mapsto p r_{1} \circ g\right|_{S^{2} \times\{p t\}} .
$$

For more details, see 1 .

The Leray-Hirsch Theorem then implies that the Leray-Serre cohomology spectral sequence for the fibration

$$
K_{k} \longrightarrow G_{\lambda} \stackrel{p}{\longrightarrow} G_{\lambda} / K_{k}
$$

collapses with

$$
E_{\infty}^{*, *} \cong E_{2}^{*, *} \cong H^{*}\left(G_{\lambda} / K_{k}\right) \otimes H^{*}\left(K_{k}\right)
$$

as bigraded algebras. This does not quite prove (2), since all it says is that the bigraded algebra $E_{0}^{*, *}\left(H^{*}\left(G_{\lambda}\right), F\right)$, associated to $H^{*}\left(G_{\lambda}\right)$ with filtration $F$ coming from the above fibration, is isomorphic to $H^{*}\left(G_{\lambda} / K_{k}\right) \otimes H^{*}\left(K_{k}\right)$. 
To finish the proof of (2) we can now do the following. $H^{*}\left(G_{\lambda}\right)$ has a subalgebra $p^{*}\left(H^{*}\left(G_{\lambda} / K_{k}\right)\right) \cong H^{*}\left(G_{\lambda} / K_{k}\right)$. Since

$$
i^{*}: H^{*}\left(G_{\lambda}\right) \rightarrow H^{*}\left(K_{k}\right)
$$

is surjective, we can choose $a, b \in H^{o d d}\left(G_{\lambda}\right)$ so that $i^{*}(a)$ and $i^{*}(b)$ generate the ring $H^{*}\left(K_{k}\right)$. The subalgebra of $H^{*}\left(G_{\lambda}\right)$ generated by $a$ and $b$ is isomorphic to $H^{*}\left(K_{k}\right)$ since the only relations in this algebra hold universally on any cohomology algebra (skew-symmetry of the cup product of elements of odd degree). Composing these inclusions of $H^{*}\left(G_{\lambda} / K_{k}\right)$ and $H^{*}\left(K_{k}\right)$ as subalgebras of $H^{*}\left(G_{\lambda}\right)$ with cup product multiplication in $H^{*}\left(G_{\lambda}\right)$ we get a map

$$
\nu: H^{*}\left(G_{\lambda} / K_{k}\right) \otimes H^{*}\left(K_{k}\right) \rightarrow H^{*}\left(G_{\lambda}\right)
$$

Because $H^{*}\left(G_{\lambda}\right)$ is (graded) commutative we have that $\nu$ is an algebra homomorphism. Moreover, by construction, $\nu$ is compatible with filtrations (the obvious one on $H^{*}\left(G_{\lambda} / K_{k}\right) \otimes H^{*}\left(K_{k}\right)$ and $F$ on $\left.H^{*}\left(G_{\lambda}\right)\right)$. As was already remarked, the degeneration of the spectral sequence at the $E_{2}$ term implies that the associated bigraded version of $\nu$ is an algebra isomorphism, which in turn shows that $\nu$ itself is an algebra isomorphism, concluding the proof of (2).

Part (ii) of the proposition is a direct consequence of (i).

Corollary 3.2. If $1 \leq k \leq \ell-1$ the link $L_{k+1}$ of $U_{k+1}$ in $U_{k}$ represents a nonzero element of $H_{3}\left(U_{k}\right)$.

Proof. By Proposition 2.9 $L_{k+1}$ is a homogeneous space for $K_{k+1}$. Hence, under the map $G_{\lambda} \rightarrow G_{\lambda} / K_{k}=U_{k}$, the generator of $H_{3}\left(K_{k+1}\right)$ is taken to the fundamental class of $H_{3}\left(L_{k+1}\right)$. Since the inclusion map $H_{3}\left(K_{k+1}\right) \rightarrow H_{3}\left(G_{\lambda}\right)$ is nonzero, the same holds for the inclusion $H_{3}\left(L_{k+1}\right) \rightarrow U_{k}$.

QED

The computation of $H^{*}\left(G_{\lambda}\right)$ is based on using the isomorphisms in (i) above and on the Mayer-Vietoris sequences for the decompositions

$$
U_{0 \ldots k}=U_{0 \ldots k-1} \cup \mathcal{N}\left(U_{k}\right),
$$

where $\mathcal{N}\left(U_{k}\right)$ is a neighborhood of $U_{k}$ in $U_{0 \ldots k}$. By Proposition 2.4 we may choose $\mathcal{N}\left(U_{k}\right)$ to be a disc bundle over $U_{k}$. In particular, there is a fibration

$$
S^{4 k-3} \rightarrow \partial \mathcal{N}\left(U_{k}\right) \rightarrow U_{k} .
$$

Lemma 3.3. For all $k \leq \ell$

$$
H^{i}\left(U_{0 \ldots k}\right)=0, \quad 0<i \leq 4 k .
$$

Proof. The Mayer-Vietoris sequence for the decomposition $U_{0 \ldots k}=U_{0 \ldots k-1} \cup$ $\mathcal{N}\left(U_{k}\right)$ is

$$
\rightarrow H^{i}\left(U_{0 \ldots k-1}\right) \oplus H^{i}\left(\mathcal{N}\left(U_{k}\right)\right) \rightarrow H^{i}\left(U_{0 \ldots k-1} \cap \mathcal{N}\left(U_{k}\right)\right) \rightarrow H^{i+1}\left(U_{0 \ldots k}\right) \rightarrow \cdots .
$$

But, when $i<4 k-3$,

$$
H^{i}\left(U_{0 \ldots k-1} \cap \mathcal{N}\left(U_{k}\right)\right)=H^{i}\left(\partial \mathcal{N}\left(U_{k}\right)\right)=H^{i}\left(\mathcal{N}\left(U_{k}\right)\right),
$$

since the fiber of the projection $\partial \mathcal{N}\left(U_{k}\right) \rightarrow U_{k}$ is connected up to dimension $4 k-3$. This implies that

$$
H^{i}\left(U_{0 \ldots k}\right) \cong H^{i}\left(U_{0 \ldots k-1}\right), \quad i \leq 4 k-4 .
$$

The lemma follows by downwards induction on $k$ because $U_{0 \ldots \ell}=\mathcal{J}_{\lambda}$ is contractible. 
The next result is contained in [1]. Its proof is repeated here for the convenience of the reader.

Proposition 3.4. (i) $H^{1}\left(U_{0}\right)=\mathbb{Q}$ and its generator is nonzero on the class in $H_{1}\left(U_{0}\right)$ represented by the link $L_{1}$ of $U_{1}$ in $U_{0}$.

(ii) There is an exact sequence

$\left(M V_{1}\right): \cdots \rightarrow H^{i-2}\left(U_{1}\right) \rightarrow H^{i}\left(U_{01}\right) \rightarrow H^{i}\left(U_{0}\right) \stackrel{\tau}{\rightarrow} H^{i-1}\left(U_{1}\right) \stackrel{\delta}{\rightarrow} H^{i+1}\left(U_{01}\right) \rightarrow \cdots$.

Proof. Using Lemma 3.3 on the Mayer-Vietoris sequence for the decomposition $U_{01}=U_{0} \cup \mathcal{N}\left(U_{1}\right)$ we get the short exact sequence:

$$
H^{1}\left(U_{01}\right)=0 \rightarrow H^{1}\left(U_{0}\right) \oplus H^{1}\left(\mathcal{N}\left(U_{1}\right)\right) \rightarrow H^{1}\left(\partial \mathcal{N}\left(U_{1}\right)\right) \rightarrow 0=H^{2}\left(U_{01}\right) .
$$

If $r=\operatorname{rk} H^{1}\left(\mathcal{N}\left(U_{1}\right)\right)=\operatorname{rk} H^{1}\left(U_{1}\right)$, it follows from Proposition 3.1 (i) that $r+$ $1=\operatorname{rk} H^{1}\left(U_{0}\right)$ and hence we get from the previous short exact sequence that $2 r+1=\operatorname{rk} H^{1}\left(\partial \mathcal{N}\left(U_{1}\right)\right)$. The existence of a fibration $S^{1} \hookrightarrow \partial \mathcal{N}\left(U_{1}\right) \rightarrow U_{1}$ shows that

$$
2 r+1=\operatorname{rk} H^{1}\left(\partial \mathcal{N}\left(U_{1}\right)\right) \leq \operatorname{rk} H^{1}\left(U_{1}\right)+1=r+1
$$

which implies that $r=0$.

We conclude that $H^{1}\left(\partial \mathcal{N}\left(U_{1}\right)\right) \cong \mathbb{Q} \cong H^{1}\left(U_{0}\right)$ and also that the inclusion $S^{1} \hookrightarrow$ $\partial \mathcal{N}\left(U_{1}\right)$ is injective in homology (or surjective in cohomology), which proves (i).

Moreover, it also follows that the cohomology spectral sequence for the fibration

$$
S^{1} \rightarrow \partial \mathcal{N}\left(U_{1}\right) \rightarrow U_{1}
$$

collapses and

$$
H^{*}\left(\partial \mathcal{N}\left(U_{1}\right)\right) \cong H^{*}\left(S^{1} \times U_{1}\right) \cong H^{*}\left(U_{1}\right) \oplus H^{*-1}\left(U_{1}\right)
$$

Part (ii) now follows easily.

QED

Corollary 3.5. $H^{2}\left(U_{0}\right)=H^{3}\left(U_{0}\right)=0$ and $H^{3}\left(U_{k}\right)=\mathbb{Q}$ for $k \geq 1$. Moreover, the smallest integer $d>3$ such that $H^{d}\left(U_{k}\right) \neq 0$ is the same for all $k \geq 0$.

Proof. Since $H^{1}\left(U_{0}\right)=\mathbb{Q}$ we have by the isomorphisms in Proposition [3.1 (i) that $H^{1}\left(U_{1}\right)=0$. Using this and the fact that $H^{i}\left(U_{01}\right)=0,0<i \leq 4$, by Lemma 3.3 . in $\left(M V_{1}\right)$ we conclude that $H^{2}\left(U_{0}\right)=H^{2}\left(U_{1}\right)=H^{3}\left(U_{0}\right)=0$. From $H^{3}\left(U_{0}\right)=0$, and again using Proposition 3.1 (i), we also get that $H^{3}\left(U_{k}\right)=\mathbb{Q}$ for $k \geq 1$. The statement about $d$ follows similarly by Proposition 3.1 (i).

QED

Proposition 3.6. For all $1 \leq k \leq \ell$ there is an exact sequence

$$
\left(M V_{k}\right): \cdots \rightarrow H^{i}\left(U_{0 \ldots k}\right) \rightarrow H^{i}\left(U_{0 \ldots k-1}\right) \stackrel{\tau}{\rightarrow} H^{i-4 k+3}\left(U_{k}\right) \stackrel{\delta}{\rightarrow} H^{i+1}\left(U_{0 \ldots k}\right) \rightarrow \cdots .
$$

Proof. Observe that because $H^{i}\left(U_{0 \ldots k}\right)=0$, for $i \leq 4 k$, the Mayer-Vietoris sequence for the pair $\left(U_{0 \ldots k}, U_{k}\right)$ implies that

$$
H^{i}\left(U_{0 \ldots k-1}\right) \oplus H^{i}\left(\mathcal{N}\left(U_{k}\right)\right) \cong H^{i}\left(\partial \mathcal{N}\left(U_{k}\right)\right), \quad i<4 k .
$$

But $\mathcal{N}\left(U_{k}\right) \simeq U_{k}$ and the fiber of the projection

$$
f: \partial \mathcal{N}\left(U_{k}\right) \rightarrow U_{k}
$$

is $S^{4 k-3}$. Therefore the fact that $H^{4 k-2}\left(U_{k}\right)$ injects into $H^{4 k-2}\left(\partial \mathcal{N}\left(U_{k}\right)\right)$ implies that all differentials in the Leray spectral sequence of $f$ vanish. Hence the map

$$
H^{i}\left(U_{k}\right) \cong H^{i}\left(\mathcal{N}\left(U_{k}\right)\right) \rightarrow H^{i}\left(\partial \mathcal{N}\left(U_{k}\right)\right)
$$


is injective for all $i$ (not just for $i<4 k$ ) and has cokernel isomorphic to $H^{i-4 k+3}\left(U_{k}\right)$. Hence $\left(M V_{k}\right)$ is exact for all $k \leq \ell$.

QED

Proposition 3.7. (i) In $\left(M V_{k}\right)$, for $k<\ell$, the boundary map

$$
\delta_{k}: \mathbb{Q}=H^{3}\left(U_{k}\right) \rightarrow H^{4 k+1}\left(U_{0 \ldots k}\right)
$$

is an isomorphism.

(ii) $H^{4 k+j}\left(U_{0 \ldots k}\right)=0$ for $j=2,3$ and $1 \leq k<\ell$. Moreover

$$
\begin{aligned}
& H^{4 \ell}\left(U_{0 \ldots \ell-1}\right)=\mathbb{Q}, \\
& H^{4 k+4}\left(U_{0 \ldots k}\right)=0, \quad k<\ell-1 .
\end{aligned}
$$

Proof. (i) From $\left(M V_{k+1}\right)$ with $i=4 k+1$ we find that

$$
H^{4 k+1}\left(U_{0 \ldots k+1}\right) \rightarrow H^{4 k+1}\left(U_{0 \ldots k}\right) \stackrel{\tau}{\rightarrow} H^{0}\left(U_{k+1}\right) \rightarrow H^{4 k+2}\left(U_{0 \ldots k+1}\right)
$$

is exact. Since the first and last terms above are 0 by Lemma 3.3 and the third is $\mathbb{Q}$, we get that $H^{4 k+1}\left(U_{0 \ldots k}\right)=\mathbb{Q}$. Moreover, the way $\left(M V_{k+1}\right)$ is constructed out of Mayer-Vietoris for $U_{0 \ldots k+1}=U_{0 \ldots k} \cup \mathcal{N}\left(U_{k+1}\right)$ and degeneration of

$$
S^{4 k+1} \rightarrow \partial \mathcal{N}\left(U_{k+1}\right) \rightarrow U_{k+1}
$$

shows that the generator of $H^{4 k+1}\left(U_{0 \ldots k}\right)$ is nonzero on the link $S^{4 k+1}$ of $U_{k+1}$ in $U_{0 \ldots k}$, and so this link is an explicit geometric generator for the homology group $H_{4 k+1}\left(U_{0 \ldots k}\right)$.

The homology version of the map $\delta_{k}: H^{3}\left(U_{k}\right) \rightarrow H^{4 k+1}\left(U_{0 \ldots k}\right)$ in $\left(M V_{k}\right)$ sends the link $S^{4 k+1}$ of $U_{k+1}$ in $U_{0 \ldots k}$ to its intersection with $U_{k}$, i.e. to the link of $U_{k+1}$ in $U_{k}$. Hence, $\delta_{k}$ is an isomorphism because, by Corollary 3.2 the link of $U_{k+1}$ in $U_{k}$ represents a nonzero element in $H_{3}\left(U_{k}\right)$.

To prove (ii), look at $\left(M V_{k+1}\right)$ with $i=4 k+j$, where $j=2,3,4$, using the facts that $H^{1}\left(U_{k}\right)=H^{2}\left(U_{k}\right)=0$ and that $\delta_{k+1}$ is an isomorphism when $k+1<\ell$. QED

Our next aim is to show that the nonzero element in $H^{4 \ell}\left(U_{0} \ldots \ell-1\right)$ restricts to give a nonzero element in $H^{4 \ell}\left(U_{0}\right)$. Moreover, we will see that this is the "first" nonzero element in $H^{*}\left(U_{k}\right)$ in degrees $*>3$. More precisely, as in Corollary 3.5 we consider the smallest integer $d=d_{\ell}>3$ such that $H^{d}\left(U_{k}\right) \neq 0$ for any $k$, and will show that $d_{\ell}=4 \ell$.

Lemma 3.8. $d_{1}=4, d_{2}=8$ while $d_{\ell}>11$ when $\ell>2$.

Proof. The fact that $d_{1}=4$ follows from Proposition 3.7 (ii).

Consider the sequence $\left(M V_{1}\right)$ with $i=d_{\ell}=d$ :

$$
\cdots \rightarrow H^{d-2}\left(U_{1}\right) \stackrel{\delta}{\rightarrow} H^{d}\left(U_{01}\right) \rightarrow H^{d}\left(U_{0}\right) \stackrel{\tau}{\rightarrow} H^{d-1}\left(U_{1}\right)=0 .
$$

Clearly, $d$ is the smallest integer $>3$ such that $H^{d}\left(U_{01}\right)$ is nonzero and also not covered by the image of $\delta$.

Now suppose that $\ell=2$. In this case the sequence $\left(M V_{2}\right)$ collapses to the isomorphisms

$$
\tau: H^{i}\left(U_{01}\right) \stackrel{\cong}{\rightarrow} H^{i-5}\left(U_{2}\right), \quad i>0 .
$$

Hence the first nonzero groups $H^{i}\left(U_{01}\right)$ (for $i>0$ ) occur when $i=5,8$. By Proposition 3.7 (i) we know that $H^{5}\left(U_{01}\right)$ is in the image of $\delta$, and so $d_{2} \geq 8$. This implies that $H^{6}\left(U_{1}\right)=0$, which means that $H^{8}\left(U_{01}\right)$ is not in the image of $\delta$. Hence $d_{2}=8$. 
If $\ell>2$ the argument showing that $d_{\ell}>5$ goes through as before. Now look at $\left(M V_{2}\right)$ :

$$
\cdots \rightarrow H^{i}\left(U_{012}\right) \rightarrow H^{i}\left(U_{01}\right) \stackrel{\tau}{\rightarrow} H^{i-5}\left(U_{2}\right) \stackrel{\delta}{\rightarrow} H^{i+1}\left(U_{012}\right) \rightarrow \cdots .
$$

We know, by Lemma [3.3, that $H^{i}\left(U_{012}\right)=0$ for $i \leq 8$. Hence $H^{i}\left(U_{01}\right)=0$ for these $i$, since in this range $H^{i-5}\left(U_{2}\right)$ is either zero or mapped isomorphically by $\delta$. Hence $d_{\ell}>8$. By Proposition 3.7)(i) we know that $H^{9}\left(U_{012}\right)=\mathbb{Q}$ is in the image of $\delta$, while Proposition [3.7)(ii) says that $H^{i}\left(U_{012}\right)=0$ for $i=10,11$ (and also 12 when $\ell>3)$. But in this range, because $H^{i-5}\left(U_{2}\right)=0$, we get from $\left(M V_{2}\right)$ that $H^{i}\left(U_{01}\right) \cong H^{i}\left(U_{012}\right)=0$. Feeding this information back into $\left(M V_{1}\right)$, we find that $d_{\ell}>11$ as claimed. In fact, if $\ell>3$ we get that $d_{\ell}>12$.

QED

More generally:

Proposition 3.9. $d_{\ell}=4 \ell$, for any $\ell \in \mathbb{N}$.

Proof. We will prove by induction on $\ell \in \mathbb{N}$ the statement:

$$
\text { for any } \ell \in \mathbb{N}, d_{\ell}=4 \ell \text { and } d_{p}>4 \ell+3 \text { for } p>\ell
$$

which implies the result and is more convenient for the inductive step.

As the previous lemma shows, the statement is true for $\ell=1,2$. We now suppose, by induction, that the statement is true for $\ell-1 \geq 2$ and will consider the case $\ell$. Combining Lemma 3.3 with Proposition 3.7 we have that

$$
\begin{aligned}
H^{i}\left(U_{0 \ldots \ell-1}\right) & =0, \quad i<4 \ell, i \neq 4 \ell-3, \\
H^{4 \ell-3}\left(U_{0 \ldots \ell-1}\right) & \cong \mathbb{Q}, \\
H^{4 \ell}\left(U_{0 \ldots \ell-1}\right) & \cong \mathbb{Q} .
\end{aligned}
$$

We will show that for all $0 \leq k<\ell-1$,

$$
\begin{aligned}
H^{i}\left(U_{0 \ldots k}\right) & =0, \quad 4 \ell-4<i<4 \ell, \\
H^{4 \ell}\left(U_{0 \ldots k}\right) & \cong \mathbb{Q} .
\end{aligned}
$$

This for $k=0$ together with the inductive hypothesis saying that $d_{\ell}>4 \ell-1$ immediately implies that $d_{\ell}=4 \ell$.

Consider the sequence $\left(M V_{k}\right)$ for $k=\ell-1, \ell-2$ and so on:

$$
\cdots \rightarrow H^{i}\left(U_{0 \ldots k}\right) \rightarrow H^{i}\left(U_{0 \ldots k-1}\right) \stackrel{\tau}{\rightarrow} H^{i-4 k+3}\left(U_{k}\right) \stackrel{\delta}{\rightarrow} H^{i+1}\left(U_{0 \ldots k}\right) \rightarrow \cdots .
$$

When $4 \ell-4<i \leq 4 \ell$ and $1 \leq k \leq \ell-1$, the numbers $i-4 k+3$ range in the interval $[4,4 \ell-1]$, and so the inductive hypothesis implies that all $H^{i-4 k+3}\left(U_{k}\right)=0$. In order to deduce that $H^{i}\left(U_{0 \ldots k}\right) \cong H^{i}\left(U_{0 \ldots k-1}\right)$, for $4 \ell-4<i \leq 4 \ell$ and $1 \leq k \leq \ell-1$, we also need to know that $H^{i-4 k+2}\left(U_{k}\right)=0$. This holds except when $i=4 \ell-3$ and $k=\ell-1$, since then $i-4 k+2=3$. However, this nonzero group exactly cancels out the nonzero group $H^{4 \ell-3}\left(U_{0 \ldots \ell-1}\right)$ via $\delta$. Hence the groups $H^{i}\left(U_{0 \ldots k}\right)=0$ for $4 \ell-4<i<4 \ell$ and $k=\ell-2$, and remain 0 as $k$ decreases to 0 . Similarly, the groups $H^{4 \ell}\left(U_{0 \ldots k}\right)=\mathbb{Q}$ for all $0 \leq k \leq \ell-1$.

Now consider the case $p-1<\lambda \leq p$ with $\ell<p \in \mathbb{N}$. In this case we know that

$$
\begin{aligned}
H^{i}\left(U_{0 \ldots \ell-1}\right) & =0, \quad i \leq 4 \ell, i \neq 4 \ell-3, \\
H^{4 \ell-3}\left(U_{0 \ldots \ell-1}\right) & =\mathbb{Q},
\end{aligned}
$$


and the previous argument shows that $d_{p}>4 \ell$. We also must show that $H^{i}\left(U_{0}\right)=0$ for $4 \ell<i \leq 4 \ell+3$. But because $p>\ell$ Proposition 3.7 says that

$$
\begin{aligned}
H^{i}\left(U_{0} \ldots \ell\right) & =0, \quad i=4 \ell+2,4 \ell+3, \\
H^{4 \ell+1}\left(U_{0 \ldots \ell}\right) & =\mathbb{Q} .
\end{aligned}
$$

If we repeat the previous argument (replacing $\ell$ by $\ell+1$ ) we find that $H^{i}\left(U_{0 \ldots k}\right)=0$ for $4 \ell+1 \leq i \leq 4 \ell+3$ and $k=\ell-1, \ell-2, \ldots, 1$. We cannot use this argument to reach $k=0$. However, as at the beginning of the proof of Lemma 3.8, it is enough to know that $H^{i}\left(U_{01}\right)=0$ for these $i$ to conclude that $d_{p}>4 \ell+3$.

QED

Definition 3.10. We define an explicit new element $w_{\ell} \in H^{4 \ell}\left(G_{\lambda}\right)$. Let $y \in$ $H^{*}\left(G_{\lambda}\right)$ come from the second $\mathrm{SO}(3)$ factor in $K_{0}$ as in Lemma 2.10, and let $y_{k} \in H^{3}\left(U_{k}\right)$ be its image under the isomorphism of Proposition 3.1. Because $U_{0 \ldots \ell} \equiv \mathcal{J}_{\lambda}$ is contractible, the map

$$
\tau: H^{4 \ell}\left(U_{0 \ldots \ell-1}\right) \longrightarrow H^{3}\left(U_{\ell}\right)
$$

in $\left(M V_{\ell}\right)$ is an isomorphism. Hence $y_{\ell}$ lifts to an element $\bar{y}_{\ell}$ in $H^{4 \ell}\left(U_{0 \ldots \ell-1}\right)$ and then, by the arguments above, restricts to a nonzero element $v_{0}$ of $H^{4 \ell}\left(U_{0}\right)$. By Proposition 3.1, there is a corresponding element in $H^{4 \ell}\left(G_{\lambda}\right)$ and it is this that is called $w_{\ell}$. When there is no possibility of confusion, we will write $w$ instead of $w_{\ell}$. Finally, for $k=1, \ldots, \ell$, we denote by $v_{k}$ the image of $w$ in $H^{4 \ell}\left(U_{k}\right)$ under the isomorphisms of Proposition 3.1

Remark 3.11. It is perhaps easier to see $\bar{y}_{\ell}$ in homology. Let $\eta$ be a spherical representative of the second $\mathrm{SO}(3)$ factor in $H_{3}\left(K_{0}\right)$, and let $S \cong S^{4 \ell-3}$ be the link of $U_{\ell}$ in $\mathcal{J}_{\lambda}=U_{0 . \ldots \ell}$. Because $K_{0}$ acts on $\mathcal{J}_{\lambda}$ by multiplication on the left, there is a well-defined $4 \ell$-cycle $\eta * S$ in $U_{0 \ldots \ell-1}$ and by definition $\bar{y}_{\ell}(\eta * S)=1$. (For more details about this notation, see Lemma 6.4.) Note that we can replace $\eta$ here by any 3 -cycle in $K_{0}$ that is nonzero in $K_{\ell}$, i.e. by anything except a multiple of $\xi+\ell^{2} \eta$. In $\$ 5$ it is natural to consider the cycle $\xi+(\ell+1)^{2} \eta$ that lives in $K_{\ell+1}$, while in $₫ 6$ we will consider $\xi$.

Since $H^{*}\left(G_{\lambda}\right)$ is the product of an exterior algebra with a polynomial algebra, we now know that

$$
\Lambda(a, x, y) \otimes S(w) \subset H^{*}\left(G_{\lambda}\right),
$$

with $\operatorname{deg} a=1, \operatorname{deg} x=\operatorname{deg} y=3$ and $\operatorname{deg} w=4 \ell$. This completes the first part of the argument. Our final task is to show that these are all the generators of $H^{*}\left(G_{\lambda}\right)$, i.e., the above inclusion is actually an equality of algebras. To do this, we first calculate all the groups and maps that occur in the part of $\left(M V_{k}\right)$ generated by this subalgebra, showing in particular that the relevant part of $H^{*}\left(U_{0 \ldots k}\right)$ is a free algebra; see Lemma 3.14. Then, we show that if $d$ is the minimal degree in which $H^{*}\left(G_{\lambda}\right)$ contains something else, there must in fact be a new element in some degree $<d$.

First we must work out the relations between the different elements $v_{k} \in H^{4 \ell}\left(U_{k}\right)$ that were defined in Definition 3.10. We just saw in the proof of the previous proposition that the restriction maps

$$
H^{i}\left(U_{0 \ldots k}\right) \rightarrow H^{i}\left(U_{0 \ldots k-1}\right)
$$

are isomorphisms when $1 \leq k \leq \ell-1$ and $4 \ell-4 \leq i \leq 4 \ell$. Hence

$$
i^{*}: H^{4 \ell}\left(U_{0 \ldots k}\right) \rightarrow H^{4 \ell}\left(U_{0}\right)
$$


is an isomorphism for $0 \leq k \leq \ell-1$, and we will denote by $v_{0}$ the generator of each $H^{4 \ell}\left(U_{0 \ldots k}\right)$ such that $i^{*}\left(v_{0}\right)=v_{0}$.

Lemma 3.12. Let $i_{k}$ denote the inclusion $U_{k} \rightarrow U_{0 \ldots k}$. Then $i_{k}^{*}\left(v_{0}\right)=v_{k}$, for $0 \leq k \leq \ell-1$.

Proof. This holds for $k=0$ by definition. To avoid confusion in the following argument, we will think of $v_{0}$ as an element of $H^{*}\left(U_{0 \ldots k}\right)$ and will write $i_{0}^{*}\left(v_{0}\right)$ for its restriction to $U_{0}$. Suppose $k>0$, and let $f: P \rightarrow G_{\lambda}$ be a $4 \ell$-dimensional cycle in $G_{\lambda}$ on which $w$ does not vanish. It follows from the construction of the isomorphisms in Proposition 3.1 that the cycle $f_{k}: P \rightarrow U_{k}$, defined by $f_{k}(p)=f(p)_{*}\left(J_{2 k}\right)$ where $J_{2 k}$ is the canonical complex structure in $U_{k}$, satisfies $v_{k}\left(f_{k}\right)=w(f) \neq 0$, for all $1 \leq k \leq \ell$. Further, $i_{0}^{*}\left(v_{0}\right)\left(f_{0}\right)=w(f)$.

Since there is a path of almost complex structures in $U_{0 \ldots k}$ joining $J_{2 k}$ to $J_{0}$, the cycles $f_{k}$ and $f_{0}$ are homotopic in $U_{0 \ldots k}$. Hence,

$$
v_{0}\left(f_{k}\right)=v_{0}\left(f_{0}\right)=i_{0}^{*}\left(v_{0}\right)\left(f_{0}\right)=w(f)=v_{k}\left(f_{k}\right) .
$$

Since $H^{4 \ell}\left(U_{k}\right) \cong \mathbb{Q}$, this implies that $i_{k}^{*}\left(v_{0}\right)=v_{k}$ as claimed.

The collapse of the spectral sequence of the fibration

$$
S^{4 k-3} \rightarrow \partial \mathcal{N}\left(U_{k}\right) \stackrel{\pi}{\rightarrow} U_{k}
$$

together with the fact that the fiber is an odd-dimensional sphere, implies that we have ring isomorphisms

$$
H^{*}\left(\partial \mathcal{N}\left(U_{k}\right)\right) \cong H^{*}\left(S^{4 k-3}\right) \otimes H^{*}\left(U_{k}\right), \quad 1 \leq k \leq \ell .
$$

Hence we know that

$$
H^{*}\left(\partial \mathcal{N}\left(U_{k}\right)\right) \supseteq \Lambda\left(\pi^{*}\left(y_{k}\right), \widehat{z}_{4 k-3}\right) \otimes S\left(\pi^{*}\left(v_{k}\right)\right), \quad 1 \leq k \leq \ell,
$$

where

(i) $y_{k} \in H^{3}\left(U_{k}\right)$ is as in Definition 3.10

(ii) $\widehat{z}_{4 k-3} \in H^{4 k-3}\left(\partial \mathcal{N}\left(U_{k}\right)\right)$ is the restriction of $z_{4 k-3} \in H^{4 k-3}\left(U_{0 \ldots k-1}\right)$, and $z_{4 k-3}$ is determined from the isomorphism

$$
\tau: H^{4 k-3}\left(U_{0 \ldots k-1}\right) \rightarrow H^{0}\left(U_{k}\right)
$$

in $\left(M V_{k}\right)$ by the condition $\tau\left(z_{4 k-3}\right)=1$.

Lemma 3.13. Let $j_{k}$ denote the inclusion $\partial \mathcal{N}\left(U_{k}\right) \rightarrow U_{0 \ldots k-1}$ and $\pi$ the projection $\partial \mathcal{N}\left(U_{k}\right) \rightarrow U_{k}$. Then:

(i) $j_{k}^{*}\left(v_{0}\right)=\pi^{*}\left(v_{k}\right)$, for $0 \leq k \leq \ell-1$;

(ii) $j_{\ell}^{*}\left(v_{0}\right)=\widehat{z}_{4 \ell-3} \cdot \pi^{*}\left(y_{\ell}\right)+c \pi^{*}\left(v_{\ell}\right)$, with $c \neq 0$.

Proof. Statement (i) follows trivially from the previous lemma. Since $v_{0}$ lives in $U_{0 \ldots k}$, its restriction to $\partial \mathcal{N}\left(U_{k}\right)$ is given by $\pi^{*}\left(i_{k}^{*} v_{0}\right)=\pi^{*}\left(v_{k}\right)$.

To prove (ii), observe first that the isomorphism $\tau: H^{4 \ell}\left(U_{0 \ldots \ell-1}\right) \rightarrow H^{3}\left(U_{\ell}\right)$ from $\left(M V_{\ell}\right)$ is the composite of the restriction map

$$
j_{\ell}^{*}: H^{*}\left(U_{0 \cdots \ell-1}\right) \rightarrow H^{*}\left(\partial \mathcal{N}\left(U_{\ell}\right)\right)
$$

with integration over the fiber of the projection

$$
\pi: \partial \mathcal{N}\left(U_{\ell}\right) \rightarrow U_{\ell}
$$


Since integration over the fiber kills the elements of $\pi^{*}\left(H^{*}\left(U_{\ell}\right)\right)$ and $\tau\left(v_{0}\right)=y_{\ell}$, we must have

$$
j_{\ell}^{*}\left(v_{0}\right)=\widehat{z}_{4 \ell-3} \cdot \pi^{*}\left(y_{\ell}\right)+c \pi^{*}\left(v_{\ell}\right) .
$$

If $c=0$, then $j_{\ell}^{*}\left(v_{0}\right)^{2}=\left(\widehat{z}_{4 \ell-3} \cdot \pi^{*}\left(y_{\ell}\right)\right)^{2}=0$ because of degree considerations. Since $j_{\ell}^{*}$ is injective (because $\tau$ is an isomorphism) this implies $\left(v_{0}\right)^{2}=0$. But this is impossible, since the restriction of $v_{0}$ to $H^{*}\left(U_{0}\right)$ is nonzero and $H^{*}\left(U_{0}\right)$ is a free algebra. Hence $c \neq 0$ as stated.

QED

Lemma 3.14. For each $k, 1 \leq k \leq \ell$, the elements $z_{4 k-3}$ and $v_{0}$ generate a free subalgebra of $H^{*}\left(U_{0 \ldots k-1}\right)$.

Proof. If $1 \leq k \leq \ell-1$ this follows since

$$
j_{k}^{*}\left(z_{4 k-3} \cdot v_{0}^{n}\right)=\widehat{z}_{4 k-3} \cdot\left(\pi^{*}\left(v_{k}\right)\right)^{n} \neq 0
$$

in $H^{*}\left(\partial \mathcal{N}\left(U_{k}\right)\right)$. For $k=\ell$ we have

$$
j_{\ell}^{*}\left(z_{4 \ell-3} \cdot v_{0}^{n}\right)=\widehat{z}_{4 \ell-3} \cdot\left[\widehat{z}_{4 \ell-3} \cdot \pi^{*}\left(y_{\ell}\right)+c \pi^{*}\left(v_{\ell}\right)\right]^{n}=c^{n} \cdot \widehat{z}_{4 \ell-3} \cdot\left[\pi^{*}\left(v_{\ell}\right)\right]^{n} \neq 0
$$

in $H^{*}\left(\partial \mathcal{N}\left(U_{\ell}\right)\right)$.

QED

Proposition 3.15. Let $1 \leq k \leq \ell-1$. The maps $i^{*}, \tau$ and $\delta$ in the exact sequence $\left(M V_{k}\right)$

$$
\cdots \rightarrow H^{i}\left(U_{0 \ldots k}\right) \stackrel{i^{*}}{\rightarrow} H^{i}\left(U_{0 \ldots k-1}\right) \stackrel{\tau}{\rightarrow} H^{i-4 k+3}\left(U_{k}\right) \stackrel{\delta}{\rightarrow} H^{i+1}\left(U_{0 \ldots k}\right) \rightarrow \cdots
$$

satisfy:

$$
\begin{array}{lll}
i^{*}\left(v_{0}^{n}\right)=v_{0}^{n}, & \tau\left(v_{0}^{n}\right)=0, & \delta\left(v_{k}^{n}\right)=0, \\
i^{*}\left(z_{4 k+1} \cdot v_{0}^{n}\right)=0, & \tau\left(z_{4 k-3} \cdot v_{0}^{n}\right)=v_{k}^{n}, & \delta\left(y_{k} \cdot v_{k}^{n}\right)=c \cdot z_{4 k+1} \cdot v_{0}^{n}, c \neq 0 .
\end{array}
$$

Proof. $\quad i^{*}$ is induced by inclusion, hence a ring homomorphism. By construction we know that $i^{*}\left(v_{0}\right)=v_{0}$. Because $\delta: H^{3}\left(U_{k}\right) \rightarrow H^{4 k+1}\left(U_{0 \ldots k}\right)$ is an isomorphism for $k<\ell$ (by Proposition $3.7(\mathrm{i})$ ), we also know that $i^{*}\left(z_{4 k+1}\right)=0$.

$\tau$ Since $i^{*}\left(v_{0}^{n}\right)=v_{0}^{n}$ we have that $\tau\left(v_{0}^{n}\right)=0$. By construction $\tau\left(z_{4 k-3}\right)=1$. Also

$$
\tau\left(z_{4 k-3} \cdot v_{0}^{n}\right)=\int_{S^{4 k-3}} \widehat{z}_{4 k-3} \cdot\left(\pi^{*}\left(v_{k}\right)\right)^{n}=v_{k}^{n} .
$$

$\delta$ Since $\tau\left(z_{4 k-3} \cdot v_{0}^{n}\right)=v_{k}^{n}$ we have that $\delta\left(v_{k}^{n}\right)=0$. As before, since $\delta: H^{3}\left(U_{k}\right) \rightarrow$ $H^{4 k+1}\left(U_{0 \ldots k}\right)$ is an isomorphism and $H^{3}\left(U_{k}\right) \cong \mathbb{Q}$, we know that $\delta\left(y_{k}\right)=$ $c \cdot z_{4 k+1}$, with $c \neq 0$.

It remains to calculate $\delta\left(y_{k} \cdot v_{k}^{n}\right)$. This is where the hypothesis $k \leq \ell-1$ comes in. We will restrict $\left(M V_{k}\right)$ to a neighborhood of $U_{k+1}$ and exploit the fact that the resulting sequence is a module over $H^{*}\left(U_{k+1}\right)$. The construction uses the fact that the $U_{k}$ form a stratification of $\mathcal{J}_{\lambda}$ with nice normal structure; see the discussion before Proposition 2.4. The neighborhoods occurring below are chosen to be fibered, with fiber equal to a cone over the relevant link.

Recall that $\left(M V_{k}\right)$ is derived from the Mayer-Vietoris sequence for the decomposition $U_{0 \ldots k}=U_{0 \ldots k-1} \cup \mathcal{N}\left(U_{k}\right)$. Intersecting all sets with $\mathcal{N}\left(U_{k+1}\right)$, we may identify $U_{0 \ldots k} \cap \mathcal{N}\left(U_{k+1}\right)$ with its boundary $\partial \mathcal{N}\left(U_{k+1}\right)$, and $\mathcal{N}\left(U_{k}\right) \cap \mathcal{N}\left(U_{k+1}\right)$ with its deformation retract $\partial \mathcal{N}_{k}\left(U_{k+1}\right)$. (Here we write $\mathcal{N}_{k}(X)$ for the neighborhood of $X$ in $U_{k}$ and $\partial \mathcal{N}_{k}(X)$ for its boundary.) Note also that

$$
Y=U_{0 \ldots k-1} \cap \mathcal{N}\left(U_{k}\right) \cap \mathcal{N}\left(U_{k+1}\right)
$$


is (rationally) homotopy equivalent to an odd-dimensional sphere bundle over $\partial \mathcal{N}_{k}\left(U_{k+1}\right)\left(S^{1}\right.$-bundle if $k=1$ and $S^{3}$-bundle if $\left.k>1\right)$.

Thus the Mayer-Vietoris sequence has the form

$$
\rightarrow H^{i}\left(\partial \mathcal{N}_{k}\left(U_{k+1}\right)\right) \oplus H^{i}\left(U_{0 \ldots k-1} \cap \mathcal{N}\left(U_{k+1}\right)\right) \rightarrow H^{i}(Y) \rightarrow H^{i+1}\left(\partial \mathcal{N}\left(U_{k+1}\right)\right) \rightarrow
$$

and, as with $\left(M V_{k}\right)$, it gives rise to an exact sequence

$$
(*) \rightarrow H^{i}\left(U_{0 \ldots k-1} \cap \mathcal{N}\left(U_{k+1}\right)\right) \stackrel{\tau^{\prime}}{\rightarrow} H^{i-4 k+3}\left(\partial \mathcal{N}_{k}\left(U_{k+1}\right)\right) \stackrel{\delta^{\prime}}{\rightarrow} H^{i+1}\left(\partial \mathcal{N}\left(U_{k+1}\right)\right) \rightarrow .
$$

All these spaces fiber over $U_{k+1}$ in a compatible way. Hence the homology groups involved are $H^{*}\left(U_{k+1}\right)$-modules, all the maps in the Mayer-Vietoris sequence preserve this module structure, and it is not hard to check that those in the modified sequence $(*)$ do as well.

Next, consider the commutative diagram:

$$
\begin{array}{ccc}
H^{i-4 k-3}\left(U_{k}\right) & \stackrel{\delta}{\rightarrow} & H^{i+1}\left(U_{0 \ldots k}\right) \\
\widehat{r}^{*} \downarrow & & \downarrow r^{*} \\
H^{i-4 k-3}\left(\partial \mathcal{N}_{k}\left(U_{k+1}\right)\right) & \stackrel{\delta^{\prime}}{\rightarrow} & H^{i+1}\left(\partial \mathcal{N}\left(U_{k+1}\right)\right)
\end{array}
$$

where the vertical maps are the obvious restrictions. Thus $r^{*}$ is the map previously called $j_{k+1}^{*}$. From the two preceding lemmas we know that $r^{*}$ is injective and

$$
r^{*}\left(\delta\left(y_{k}\right)\right)=c r^{*}\left(z_{4 k+1}\right)=c \widehat{z}_{4 k+1}, \quad c \neq 0 .
$$

Defining $\widehat{y}_{k}=\widehat{r}^{*}\left(y_{k}\right)$, and using commutativity of the diagram, we have that $\left.\delta^{\prime}\left(\widehat{y}_{k}\right)\right)=c \widehat{z}_{4 k+1}$.

In order to compute $\delta^{\prime} \circ \widehat{r}^{*}\left(y_{k} \cdot v_{k}^{n}\right)$ we will show that $\widehat{r}^{*}\left(v_{k}\right)$ is pulled back from $H^{*}\left(U_{k+1}\right)$ via the projection

$$
\pi: \partial \mathcal{N}_{k}\left(U_{k+1}\right) \longrightarrow U_{k+1},
$$

and then will use the module property. Consider the commutative diagram of inclusions:

$$
\begin{array}{ccc}
\partial \mathcal{N}\left(U_{k+1}\right) & \stackrel{r}{\rightarrow} & U_{0 \ldots k} \\
i_{\partial} \uparrow & & \uparrow i_{k} \\
\partial \mathcal{N}_{k}\left(U_{k+1}\right) & \stackrel{\widehat{r}}{\rightarrow} & U_{k}
\end{array}
$$

From Lemmas 3.12 and 3.13 we have that for $1 \leq k \leq \ell-2$

$$
\widehat{r}^{*}\left(v_{k}\right)=\widehat{r}^{*} \circ i_{k}^{*}\left(v_{0}\right)=i_{\partial}^{*} \circ r^{*}\left(v_{0}\right)=i_{\partial}^{*} \circ \pi^{*}\left(v_{k+1}\right) .
$$

(Recall that $r=j_{k+1}$.) On the other hand, for $k=\ell-1$,

$$
\widehat{r}^{*}\left(v_{\ell-1}\right)=i_{\partial}^{*} \circ r^{*}\left(v_{0}\right)=i_{\partial}^{*}\left(\widehat{z}_{4 \ell-3} \cdot \pi^{*}\left(y_{\ell}\right)+c \pi^{*}\left(v_{\ell}\right)\right), \quad c \neq 0 .
$$

If we define

$$
\widehat{\pi}=\pi \circ i_{\partial}: \partial \mathcal{N}_{k}\left(U_{k+1}\right) \rightarrow U_{k+1},
$$

this can be written as

$$
\widehat{r}^{*}\left(v_{k}\right)=\widehat{\pi}^{*}\left(v_{k+1}\right) \text { for } 1 \leq k \leq \ell-2,
$$

while

$$
\widehat{r}^{*}\left(v_{\ell-1}\right)=i_{\partial}^{*}\left(\widehat{z}_{4 \ell-3}\right) \cdot \widehat{\pi}^{*}\left(y_{\ell}\right)+c \cdot \widehat{\pi}^{*}\left(v_{\ell}\right) .
$$

We saw in Proposition 2.4 that the fiber $L_{\ell}$ of the fibration

$$
L_{\ell} \hookrightarrow \partial \mathcal{N}_{\ell-1}\left(U_{\ell}\right) \stackrel{\widehat{\pi}}{\rightarrow} U_{\ell}
$$


is a 3-dimensional lens space. Moreover the Leray-Serre spectral sequence collapses by Corollary 3.2. Hence

$$
H^{4 \ell}\left(\partial \mathcal{N}_{\ell-1}\left(U_{\ell}\right)\right) \cong \widehat{\pi}^{*}\left(H^{4 \ell}\left(U_{\ell}\right)\right) \oplus H^{4 \ell-3}\left(U_{\ell}\right) \cong H^{4 \ell}\left(U_{\ell}\right)
$$

by Proposition 3.9, Thus

$$
\widehat{r}^{*}\left(v_{\ell-1}\right)=c \cdot \widehat{\pi}^{*}\left(v_{\ell}\right) .
$$

We can now use the fact that $\delta^{\prime}$ is a map of $H^{*}\left(U_{k+1}\right)$-modules to conclude that for $1 \leq k \leq \ell-2$

$$
\begin{aligned}
\delta^{\prime} \circ \widehat{r}^{*}\left(y_{k} \cdot v_{k}^{n}\right) & =\delta^{\prime}\left(\widehat{y}_{k}\left(\widehat{r}^{*}\left(v_{k}\right)\right)^{n}\right)=\delta^{\prime}\left(\widehat{y}_{k} \widehat{\pi}^{*}\left(v_{k+1}\right)^{n}\right) \\
& =\pi^{*}\left(v_{k+1}\right)^{n} \cdot \delta^{\prime}\left(\widehat{y}_{k}\right)=r^{*}\left(v_{0}\right)^{n} \cdot c \cdot \widehat{z}_{4 k+1} \\
& =r^{*}\left(c \cdot z_{4 k+1} \cdot v_{0}^{n}\right),
\end{aligned}
$$

while for $k=\ell-1$

$$
\delta^{\prime} \circ \widehat{r}^{*}\left(y_{\ell-1} \cdot v_{\ell-1}^{n}\right)=r^{*}\left(c^{n} c \cdot z_{4 \ell-3} \cdot v_{0}^{n}\right) .
$$

Therefore,

$$
\delta\left(y_{k} \cdot v_{k}^{n}\right)=c \cdot z_{4 k+1} \cdot v_{0}^{n}, \quad c \neq 0
$$

for all $1 \leq k \leq \ell-1$, as claimed.

QED

Proof of Theorem 1.1. Suppose that

$$
H^{*}\left(G_{\lambda}\right) \neq A=\Lambda(a, x, y) \otimes S(w)
$$

and let

$$
u \in H^{*}\left(G_{\lambda}\right)-A
$$

be a nonzero element of minimal degree $d$. From the arguments leading to Proposition 3.9 and its proof we know that $d>4 \ell$. The existence of this new element $u$ gives rise to new nonzero elements

$$
u_{k} \in H^{d}\left(U_{0 \ldots k}\right)-\left(\Lambda\left(z_{4 k+1}\right) \otimes S\left(v_{0}\right)\right),
$$

for $0 \leq k \leq \ell-1$, in the following inductive way. For $k=0$ we let $u_{0}$ be the image of $u$ under the relevant isomorphism from Proposition 3.1 (i). Given a nonzero element

$$
u_{k-1} \in H^{d}\left(U_{0 \ldots k-1}\right)-\left(\Lambda\left(z_{4 k-3}\right) \otimes S\left(v_{0}\right)\right), \quad 1 \leq k \leq \ell-1,
$$

consider the following piece of $\left(M V_{k}\right)$ :

$$
\cdots \rightarrow H^{d}\left(U_{0 \ldots k}\right) \stackrel{i^{*}}{\rightarrow} H^{d}\left(U_{0 \ldots k-1}\right) \stackrel{\tau}{\rightarrow} H^{d-4 k+3}\left(U_{k}\right) \stackrel{\delta}{\rightarrow} H^{d+1}\left(U_{0 \ldots k}\right) \rightarrow \cdots
$$

By exactness we know that that $\tau\left(u_{k-1}\right) \in \operatorname{Ker} \delta$, and by Proposition 3.15 and minimality of $d$ this implies that

$$
\tau\left(U_{k-1}\right)=v_{k}^{n}=\tau\left(z_{4 k-3} \cdot v_{0}^{n}\right)
$$

for some $n$. Hence the nonzero element $u_{k-1}-z_{4 k-3} \cdot v_{0}^{n}$ belongs to $\operatorname{Ker} \tau$ and, using exactness again, there must exist a nonzero $u_{k} \in H^{d}\left(U_{0 \ldots k}\right)$ such that

$$
i^{*}\left(u_{k}\right)=u_{k-1}-z_{4 k-3} \cdot v_{0}^{n} .
$$

It is clear from Proposition 3.15 that $u_{k} \notin \Lambda\left(z_{4 k+1}\right) \otimes S\left(v_{0}\right)$. 
The element $u_{\ell-1} \in H^{d}\left(U_{0 \ldots \ell-1}\right)-\left(\Lambda\left(z_{4 \ell-3}\right) \otimes S\left(v_{0}\right)\right)$ determines, via $\left(M V_{\ell}\right)$ and the fact that $U_{0 \ldots \ell}$ is contractible, a new nonzero element

$$
u_{\ell} \in H^{d-4 \ell+3}\left(U_{\ell}\right)-\left(\Lambda\left(y_{\ell}\right) \otimes S\left(v_{\ell}\right)\right) \text {. }
$$

But this is impossible, since it would give rise to a nonzero element in $H^{*}\left(G_{\lambda}\right)-A$ of degree $d-4 \ell+3$, contradicting the minimality of $d$.

3.2. Computation of $H^{*}\left(G_{\lambda}^{1}\right)$. Throughout, we fix $\ell \in \mathbb{N}$ and $\lambda \in \mathbb{R}$ such that $\ell-1<\lambda \leq \ell$. Recall that, for each $0 \leq k \leq \ell$, the stratum $U_{k}^{1}$ consists of all almost complex structures $J \in \mathcal{J}_{\lambda}^{1}$ for which the class $E_{2 k+1}$ has a $J$-holomorphic representative, and $K_{k}^{1} \cong \mathrm{U}(2)$ denotes the group of Kähler isometries of a standard complex structure $J_{2 k+1} \in U_{k}^{1}$. Moreover, as was shown in $2.4, G_{\lambda}^{1} / K_{k}^{1}$ is weakly homotopy equivalent to the strata $U_{k}^{1}$.

This whole subsection is devoted to $M_{\lambda}^{1}=\left(\mathbb{C} P^{2} \# \overline{\mathbb{C} P^{2}}, \omega_{\lambda}^{1}\right)$ and the computation of $H^{*}\left(G_{\lambda}^{1}\right)$, a process which is completely analogous to what we did in the previous subsection for $H^{*}\left(G_{\lambda}^{0}\right)$. To avoid unnecessary repetitions, we will just state the relevant lemmas, propositions and corollaries, leaving all the proofs as exercises for the interested reader. In order to simplify notation, we will also omit the superscript ${ }^{1}$.

Proposition 3.16. (i) $H^{*}\left(G_{\lambda}\right)$ is a free algebra. Moreover

$$
H^{*}\left(G_{\lambda}\right) \cong H^{*}\left(U_{k}\right) \otimes H^{*}\left(K_{k}\right), \text { for all } 0 \leq k \leq \ell,
$$

as graded algebras.

(ii) For all $0 \leq k \leq \ell, H^{*}\left(U_{k}\right) \cong H^{*}\left(U_{0}\right)$.

The computation of $H^{*}\left(G_{\lambda}\right)$ is again based on using the isomorphisms in (i) above and on the Mayer-Vietoris sequences for the decompositions

$$
U_{0 \ldots k}=U_{0 \ldots k-1} \cup \mathcal{N}\left(U_{k}\right),
$$

where $\mathcal{N}\left(U_{k}\right)$ is a neighborhood of $U_{k}$ in $U_{0 \ldots k}$. By Proposition 2.4 we may choose $\mathcal{N}\left(U_{k}\right)$ to be a disc bundle over $U_{k}$, which in particular means that there is a fibration

$$
S^{4 k-1} \rightarrow \partial \mathcal{N}\left(U_{k}\right) \rightarrow U_{k}
$$

Lemma 3.17. For all $k \leq \ell$

$$
H^{i}\left(U_{0 \ldots k}\right)=0, \quad 0<i \leq 4 k+2 .
$$

Proposition 3.18. For all $1 \leq k \leq \ell$ there is an exact sequence

$$
\left(M V_{k}\right): \cdots \rightarrow H^{i}\left(U_{0 \ldots k}\right) \rightarrow H^{i}\left(U_{0 \ldots k-1}\right) \stackrel{\tau}{\rightarrow} H^{i-4 k+1}\left(U_{k}\right) \stackrel{\delta}{\rightarrow} H^{i+1}\left(U_{0 \ldots k}\right) \rightarrow \cdots .
$$

From $\left(M V_{1}\right)$ and part (ii) of Proposition 3.16, we immediately get the following

Corollary 3.19. $H^{1}\left(U_{k}\right)=H^{2}\left(U_{k}\right)=H^{4}\left(U_{k}\right)=H^{5}\left(U_{k}\right)=0$ and $H^{3}\left(U_{k}\right) \cong \mathbb{Q}$, for all $0 \leq k \leq \ell$.

Proposition 3.20. (i) In $\left(M V_{k}\right)$, for $k<\ell$, the boundary map

$$
\delta_{k}: \mathbb{Q}=H^{3}\left(U_{k}\right) \rightarrow H^{4 k+3}\left(U_{0 \ldots k}\right)
$$

is an isomorphism.

(ii) If $1 \leq k<\ell$ the groups $H^{4 k+2+j}\left(U_{0 \ldots k}\right)=0$ for $j=2,3$. Moreover

$$
\begin{aligned}
H^{4 \ell+2}\left(U_{0 \ldots \ell-1}\right) & =\mathbb{Q}, \\
H^{4 k+6}\left(U_{0 \ldots k}\right) & =0, \quad k<\ell-1 .
\end{aligned}
$$


Denote by $d$ the smallest integer greater than 5 such that $H^{d}\left(U_{k}\right) \neq 0$. Although independent of $k$ (by Proposition $[3.16$ ), the value of $d$ depends on the integer $\ell \in \mathbb{N}$ that was fixed at the beginning of this subsection, and we make that dependence explicit by writing $d_{\ell}$.

Proposition 3.21. $d_{\ell}=4 \ell+2$, for any $\ell \in \mathbb{N}$.

Since $H^{*}\left(G_{\lambda}\right)$ is the product of an exterior algebra with a polynomial algebra, we now know that

$$
\Lambda(a, x, y) \otimes S(w) \subset H^{*}\left(G_{\lambda}\right)
$$

with $\operatorname{deg} a=1, \operatorname{deg} x=\operatorname{deg} y=3$ and $\operatorname{deg} w=4 \ell+2$. Here we define $w$ as in Definition 3.10. Our final task is to show that these are all the generators of $H^{*}\left(G_{\lambda}\right)$, i.e. the above inclusion is actually an equality of algebras.

For $k=0,1, \ldots, \ell$, let $v_{k} \in H^{4 \ell+2}\left(U_{k}\right)$ denote the generator corresponding to $w \in H^{*}\left(G_{\lambda}\right)$ under the isomorphisms of Proposition 3.16. As in the previous subsection, it follows from the proof of the previous proposition that the restriction maps

$$
H^{i}\left(U_{0 \ldots k}\right) \rightarrow H^{i}\left(U_{0 \ldots k-1}\right)
$$

are isomorphisms when $1 \leq k \leq \ell-1$ and $4 \ell-2 \leq i \leq 4 \ell+2$. Hence

$$
i^{*}: H^{4 \ell+2}\left(U_{0 \ldots k}\right) \rightarrow H^{4 \ell+2}\left(U_{0}\right)
$$

is an isomorphism for $0 \leq k \leq \ell-1$, and we also denote by $v_{0}$ the generator of each $H^{4 \ell+2}\left(U_{0 \ldots k}\right)$ such that $i^{*}\left(v_{0}\right)=v_{0}$.

Lemma 3.22. Let $i_{k}$ denote the inclusion $U_{k} \rightarrow U_{0 \ldots k}$. Then $i_{k}^{*}\left(v_{0}\right)=v_{k}$, for $0 \leq k \leq \ell-1$.

The collapse of the spectral sequence of the fibration

$$
S^{4 k-1} \rightarrow \partial \mathcal{N}\left(U_{k}\right) \stackrel{\pi}{\rightarrow} U_{k}
$$

together with the fact that the fiber is an odd-dimensional sphere, implies that we have ring isomorphisms

$$
H^{*}\left(\partial \mathcal{N}\left(U_{k}\right)\right) \cong H^{*}\left(S^{4 k-1}\right) \otimes H^{*}\left(U_{k}\right), \quad 1 \leq k \leq \ell .
$$

Hence we know that

$$
H^{*}\left(\partial \mathcal{N}\left(U_{k}\right)\right) \supseteq \Lambda\left(\pi^{*}\left(y_{k}\right), \widehat{z}_{4 k-1}\right) \otimes S\left(\pi^{*}\left(v_{k}\right)\right), \quad 1 \leq k \leq \ell,
$$

where the elements $y_{k} \in H^{3}\left(U_{k}\right)$ and $\widehat{z}_{4 k-1} \in H^{4 k-1}\left(\partial \mathcal{N}\left(U_{k}\right)\right)$ are as before. Thus the $y_{k}$ are defined as in Definition [3.10, and $\widehat{z}_{4 k-1}$ is the restriction of $z_{4 k-1} \in H^{4 k-1}\left(U_{0 \ldots k-1}\right)$, where $z_{4 k-1}$ is determined from the isomorphism $\tau$ : $H^{4 k-1}\left(U_{0 \ldots k-1}\right) \rightarrow H^{0}\left(U_{k}\right)$ in $\left(M V_{k}\right)$ by the condition $\tau\left(z_{4 k-1}\right)=1$.

Lemma 3.23. Let $j_{k}$ denote the inclusion $\partial \mathcal{N}\left(U_{k}\right) \rightarrow U_{0 \ldots k-1}$ and $\pi$ the projection $\partial \mathcal{N}\left(U_{k}\right) \rightarrow U_{k}$. Then $j_{k}^{*}: H^{*}\left(U_{0 \ldots k-1}\right) \rightarrow H^{*}\left(\partial \mathcal{N}\left(U_{k}\right)\right)$ satisfies:

(i) $j_{k}^{*}\left(v_{0}\right)=\pi^{*}\left(v_{k}\right)$, for $0 \leq k \leq \ell-1$;

(ii) $j_{\ell}^{*}\left(v_{0}\right)=\widehat{z}_{4 \ell-1} \cdot \pi^{*}\left(y_{\ell}\right)+c \pi^{*}\left(v_{\ell}\right)$, with $c \neq 0$.

Lemma 3.24. $z_{4 k-1}$ and $v_{0}$ generate free subalgebras of $H^{*}\left(U_{0 \ldots k-1}\right)$, for $1 \leq k$ $\leq \ell$. 
Proposition 3.25. In the exact sequence $\left(M V_{k}\right)$

$$
\cdots \rightarrow H^{i}\left(U_{0 \ldots k}\right) \stackrel{i^{*}}{\rightarrow} H^{i}\left(U_{0 \ldots k-1}\right) \stackrel{\tau}{\rightarrow} H^{i-4 k+1}\left(U_{k}\right) \stackrel{\delta}{\rightarrow} H^{i+1}\left(U_{0 \ldots k}\right) \rightarrow \cdots
$$

(for $1 \leq k \leq \ell-1$ ), the maps $i^{*}, \tau$ and $\delta$ satisfy:

$$
\begin{array}{lll}
i^{*}\left(v_{0}^{n}\right)=v_{0}^{n}, & \tau\left(v_{0}^{n}\right)=0, & \delta\left(v_{k}^{n}\right)=0, \\
i^{*}\left(z_{4 k+3} \cdot v_{0}^{n}\right)=0, & \tau\left(z_{4 k-1} \cdot v_{0}^{n}\right)=v_{k}^{n}, & \delta\left(y_{k} \cdot v_{k}^{n}\right)=c \cdot z_{4 k+3} \cdot v_{0}^{n}, c \neq 0 .
\end{array}
$$

The final argument to show that

$$
H^{*}\left(G_{\lambda}\right)=\Lambda(a, x, y) \otimes S(w)
$$

is exactly the same as in the previous subsection. More precisely, if

$$
H^{*}\left(G_{\lambda}\right) \neq A=\Lambda(a, x, y) \otimes S(w)
$$

then we can choose a nonzero element $u$ of minimal degree $d$ in $H^{*}\left(G_{\lambda}\right)-A$, and show that this new element $u$ would give rise to a new nonzero element in $H^{*}\left(G_{\lambda}\right)-A$ of degree $d-4 \ell+1$, which by minimality of $d$ is a contradiction.

This completes the proof of Theorem 1.4

\section{The LIMIT AT INFINITY}

In this section we consider the limit structure of the groups $G_{\lambda}^{i}$ as $\lambda \rightarrow \infty$. Our aim is to show that this limit exists and can be identified (up to homotopy) with the group $\mathcal{D}$ of fiberwise diffeomorphisms considered in \$2.5. To begin with we will consider only the case $i=0$ and, as usual, will omit superscripts. However, as we will see in Remark 4.7, our arguments apply to any ruled surface.

In order to consider the limit $\lim _{\lambda \rightarrow \infty} G_{\lambda}$, we need to construct maps $G_{\lambda} \rightarrow G_{\lambda+\kappa}$ for $\kappa>0$. However, there is no very direct way to do this, and the best approach is rather to include $G_{\lambda}$ into a space $G_{[\lambda, \lambda+\kappa]}$ that deformation retracts onto $G_{\lambda+\kappa}$. Therefore we make the following definitions.

Definition 4.1. Given a family $H_{\lambda}, \lambda \in[0, \infty)$, of subgroups of $\operatorname{Diff}\left(S^{2} \times S^{2}\right)$ that varies smoothly with $\lambda$, define

$$
\begin{aligned}
H_{\infty} & =\bigcup_{\lambda>0}\left\{(\phi, \lambda): \phi \in H_{\lambda}\right\} \subset \operatorname{Diff}\left(S^{2} \times S^{2}\right) \times \mathbb{R}^{+}, \\
H_{[\lambda, \lambda+\kappa]} & =\bigcup_{\lambda \leq \mu \leq \lambda+\kappa}\left\{(\phi, \mu): \phi \in H_{\mu}\right\} \subset \operatorname{Diff}\left(S^{2} \times S^{2}\right) \times \mathbb{R}^{+} .
\end{aligned}
$$

Our first aim is to prove the following result, which shows that

$$
G_{\infty}=\lim _{\lambda \rightarrow \infty} G_{[0, \lambda]}
$$

is the homotopy limit of the $G_{\lambda}$ as $\lambda \rightarrow \infty$.

Proposition 4.2. The inclusion $G_{\lambda+\kappa} \rightarrow G_{[\lambda, \lambda+\kappa]}$ is a weak homotopy equivalence. $G_{\lambda}$.

To prove this proposition we must consider various enlargements of the groups

Definition 4.3. We define $\widetilde{G}_{\lambda}$ to be the set of all pairs $\left(\phi, \tau_{t}\right)$, where $\phi \in$ $\operatorname{Difff}^{+}\left(S^{2} \times S^{2}\right)$ and $\tau_{t}, 0 \leq t \leq 1$, is a smooth family of cohomologous symplectic forms such that $\tau_{t}=\omega_{\lambda}$ for $t$ near 0 and $\tau_{t}=\phi^{*}\left(\omega_{\lambda}\right)$ for $t$ near 1 . Define an operation $\widetilde{G}_{\lambda} \times \widetilde{G}_{\lambda} \rightarrow \widetilde{G}_{\lambda}$ by

$$
\left(\phi, \tau_{t}\right) \circ\left(\phi^{\prime}, \tau_{t}^{\prime}\right)=\left(\phi \circ \phi^{\prime},\left(\tau * \tau^{\prime}\right)_{t}\right),
$$


where

$$
\left(\tau * \tau^{\prime}\right)_{t}= \begin{cases}\tau_{2 t}^{\prime} & \text { if } t \in[0,1 / 2] \\ \left(\phi^{\prime}\right)^{*} \tau_{2 t-1} & \text { if } t \in[1 / 2,1]\end{cases}
$$

With this operation, $\widetilde{G}_{\lambda}$ is a homotopy associative $H$-space with homotopy identity and homotopy inverse. Moreover, $G_{\lambda}$ can be identified with the subgroup of $\widetilde{G}_{\lambda}$ on which the path $\tau_{t}$ is constant.

The next lemma follows immediately from the usual Moser argument.

Lemma 4.4. $\widetilde{G}_{\lambda}$ deformation retracts onto its subgroup $G_{\lambda}$.

We would like to define a family of maps $G_{\lambda} \rightarrow \widetilde{G}_{\lambda+\kappa}$. However, as we shall see, we have to do something a little more elaborate. The next lemma will be useful.

Lemma 4.5. Let $\pi: X \rightarrow \Sigma$ be a smooth fibration of an oriented 4-manifold $X$ over an oriented 2-dimensional base manifold, and suppose that $\tau$ is a symplectic form on $X$ whose restriction to each fiber is nondegenerate. Then, if $\beta$ is any nonnegative form on $\Sigma$, the form $\tau+\pi^{*}(\beta)$ is symplectic.

Proof. For each $x \in X$ let $H_{x} \subset T_{x} X$ be the 2-dimensional $\tau$-orthogonal complement to the tangent space ker $d \pi(x)$ to the fiber at $x$. An easy calculation shows that a form $\rho$ must be nondegenerate if it restricts to $\tau$ on the fibers and is a positive multiple of $\tau$ on each $H_{x}$.

QED

Let $\mathcal{S}_{\lambda}$ denote the space of symplectic forms on $S^{2} \times S^{2}$ that are isotopic to $\omega_{\lambda}$, let Fol $_{\lambda}$ be the space of smooth foliations of $S^{2} \times S^{2}$ whose leaves are $\omega_{\lambda^{-}}$ symplectic spheres in class $F$, and let $\operatorname{Proj}_{\lambda}$ be the space of smooth surjections $p: S^{2} \times S^{2} \rightarrow S^{2}$ whose fibers form an element of $\mathrm{Fol}_{\lambda}$ and that are compatible with the obvious orientations. Then there is a fibration

$$
\operatorname{Diff}^{+}\left(S^{2}\right) \rightarrow \operatorname{Proj}_{\lambda} \rightarrow \mathrm{Fol}_{\lambda}
$$

Moreover, $\operatorname{Diff}^{+}\left(S^{2}\right)$ acts on the left on $\operatorname{Proj}_{\lambda}$ via $\psi \cdot p=\psi \circ p$, and the induced map

$$
\mathrm{SO}(3) \backslash \operatorname{Proj}_{\lambda} \rightarrow \mathrm{Fol}_{\lambda}
$$

is a homotopy equivalence. The preceding lemma implies that there is a family of maps

$$
s_{\kappa}: \operatorname{Proj}_{\lambda} \rightarrow \mathcal{S}_{\lambda+\kappa}
$$

given by

$$
s_{\kappa}(p)=\omega_{\lambda}+\kappa p^{*}(\sigma),
$$

where $\sigma$ is the usual $\mathrm{SO}(3)$-invariant area form on $S^{2}$ of total area 1 . Since $\sigma$ is $\mathrm{SO}(3)$-invariant, we in fact have a map

$$
s_{\kappa}: \mathrm{SO}(3) \backslash \operatorname{Proj}_{\lambda} \rightarrow \mathcal{S}_{\lambda+\kappa} .
$$

Lemma 4.6. The map

$$
\Psi: \mathcal{J}_{\lambda} \rightarrow \mathrm{Fol}_{\lambda}
$$

that takes $J$ to the foliation by $J$-holomorphic spheres in class $F$ is a homotopy equivalence. 
Proof. This holds by standard arguments because the inverse image of each point in $\mathrm{Fol}_{\lambda}$ is contractible.

QED

Each of the spaces $\mathcal{S}_{\lambda}, \mathrm{Fol}_{\lambda}, \operatorname{Proj}_{\lambda}$ has an obvious base point. If $Y$ is any based space we write $\mathbf{P}_{*}(Y)$ for the space of smooth paths in $Y$ starting at the base point.

The choice of a deformation retraction of $\mathcal{J}_{\lambda}$ onto the split complex structure $J_{0}$ defines a map $\iota_{r}: \mathcal{J}_{\lambda} \rightarrow \mathbf{P}_{*}\left(\mathcal{J}_{\lambda}\right)$ and hence a map

$$
G_{\lambda} \rightarrow \mathbf{P}_{*}\left(\mathrm{Fol}_{\lambda}\right): \phi \mapsto \phi^{*}\left(J_{0}\right) \mapsto \iota_{r}\left(\phi^{*}\left(J_{0}\right)\right) \mapsto \Psi_{*}\left(\iota_{r}\left(\phi^{*}\left(J_{0}\right)\right)\right)
$$

Thus there is a diagram

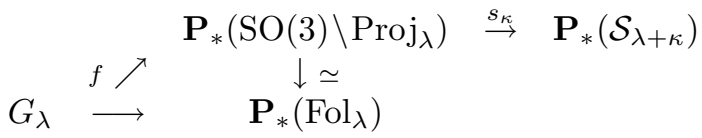

in which the lifting $f: G_{\lambda} \rightarrow \mathbf{P}_{*}\left(\mathrm{SO}(3) \backslash \operatorname{Proj}_{\lambda}\right)$ is really defined only over compact subsets of $G_{\lambda}$. Thus for each compact subset $C$ of $G_{\lambda}$ we have a map

$$
f_{C}: C \rightarrow \widetilde{G}_{\lambda+\kappa}: \quad \phi \mapsto\left(\phi, s_{\kappa}(f(\phi))\right) .
$$

Moreover, this set of maps $f_{C}$ is compatible in the sense that if $C_{1} \subset C_{2}$, then the restriction of $f_{C_{2}}$ to $C_{1}$ is homotopic to $f_{C_{1}}$.

Proof of Proposition 4.2. This follows immediately from the preceding discussion.

QED

Our next aim is to show that $G_{\infty}$ is homotopy equivalent to the group $\mathcal{D}$ of orientation preserving fiberwise diffeomorphisms, which up to homotopy is the same as

$$
\mathcal{D}=\mathrm{SO}(3) \times \operatorname{Map}\left(S^{2}, \mathrm{SO}(3)\right) .
$$

Since the elements $\phi \in \mathcal{D}$ preserve the fibers of the standard fibration $\pi: S^{2} \times S^{2} \rightarrow$ $S^{2}$, Lemma 4.5 implies that the forms $\phi^{*}\left(\omega_{\lambda}\right)+\kappa \pi^{*}(\sigma)$ are symplectic for all $\kappa$. Moreover, for sufficiently large $\kappa$ the elements of the linear isotopy

$$
t\left(\phi^{*}\left(\omega_{\lambda}\right)+\kappa \pi^{*}(\sigma)\right)+(1-t) \omega_{\lambda+\kappa}, \quad 0 \leq t \leq 1,
$$

are also all symplectic. Thus, if $\mathcal{D}_{K}$ is the subset of $\mathcal{D}$ consisting of elements whose first derivative is uniformly bounded by $K$, there is, for some constant $\kappa(K)$ a natural map

$$
\mathcal{D}_{K} \rightarrow \widetilde{G}_{\lambda+\kappa}, \quad \kappa \geq \kappa(K) .
$$

Hence there is a map from the homotopy limit

$$
\mathcal{D}^{\prime}=\lim _{\rightarrow} \mathcal{D}_{K}
$$

to $\widetilde{G}_{\infty}$. The obvious map $\mathcal{D}^{\prime} \rightarrow \mathcal{D}$ is a weak homotopy equivalence, and hence a homotopy equivalence since both spaces have the homotopy types of CW complexes. Thus we may define a map $\mathcal{D} \rightarrow G_{\infty}$ via the composite

$$
\mathcal{D} \rightarrow \mathcal{D}^{\prime} \rightarrow \widetilde{G}_{\infty} \rightarrow G_{\infty}
$$

To get a map the other way round, we must enlarge the group $\mathcal{D}$. For each $\lambda$, let

$$
\mathcal{D}_{\lambda}^{\text {Fol }}=\left\{\phi \in \operatorname{Diff}^{+}\left(S^{2} \times S^{2}\right): \phi\left(\{p\} \times S^{2}\right) \text { is } \omega_{\lambda} \text {-symplectic for all } p \in S^{2}\right\} .
$$


One has a natural fibration

$$
\mathcal{D} \rightarrow \mathcal{D}_{\lambda}^{\mathrm{Fol}} \rightarrow \mathrm{Fol}_{\lambda}
$$

Hence $\mathcal{D} \simeq \mathcal{D}_{\lambda}^{\text {Fol }}$ for all $\lambda$, since Fol $_{\lambda}$ is contractible by Lemma 4.6. Thus, defining $\mathcal{D}_{\infty}^{\text {Fol }}$ as in Definition 4.1 , we have

$$
\mathcal{D} \simeq \mathcal{D}_{\infty}^{\mathrm{Fol}}
$$

Since $G_{\infty} \subset \mathcal{D}_{\infty}^{\text {Fol }}$, we can define a map $G_{\infty} \rightarrow \mathcal{D}$ as the composite

$$
G_{\infty} \rightarrow \mathcal{D}_{\infty}^{\mathrm{Fol}} \stackrel{\simeq}{\longrightarrow} \mathcal{D}
$$

This is the homotopy inverse to the map $\mathcal{D} \rightarrow G_{\infty}$ defined before because every compact subset of $\mathcal{D}_{\lambda}^{\text {Fol }}$ is contained in $\widetilde{G}_{\lambda+\kappa}$ for suitably large $\kappa$ (the argument is the same as for $\mathcal{D}_{\lambda}$ since Lemma 4.5 can be applied with $\tau=\phi^{*}\left(\omega_{\lambda}\right)$ for any $\left.\phi \in \mathcal{D}_{\lambda}^{\mathrm{Fol}}\right)$.

Remark 4.7. The analog of this result is true for any ruled surface $X$, i.e. the limit, when the ratio of the size of the base to that of the fiber goes to infinity, of the group of symplectomorphisms of $X$ is homotopy equivalent to the group of fiberwise diffeomorphisms $\mathcal{D}(X)$ of $X$ that act trivially on $H_{2}(X)$. When $X$ is the product $\Sigma \times S^{2}$, this group $\mathcal{D}(X)$ has the homotopy type of the product $\operatorname{Diff}^{+}(\Sigma) \times \operatorname{Map}(\Sigma, \mathrm{SO}(3))$. In the case of the nontrivial bundle, let $\Gamma(\Sigma ; \mathrm{SO}(3))$ denote the space of sections of the bundle over $\Sigma$ with fiber $\mathrm{SO}(3)$ that is associated to $X$. Note first that every area-preserving diffeomorphism $\phi_{\Sigma}$ of $\Sigma$ does lift to $X$. If $\phi_{\Sigma}$ is Hamiltonian, it is the time 1 map of the flow generated by the Hamiltonian $H_{t}$, and a lift is given by the time 1 map of $H_{t} \circ \pi$. A similar argument works in general, since every area-preserving diffeomorphism of $\Sigma$ is the time 1 map of the flow generated by some family $\alpha_{t}$ of closed 1-forms on $\Sigma$. When $X \rightarrow S^{2}$ is the nontrivial $S^{2}$-bundle over $S^{2}$ we worked out the homotopy type of the relevant group $\mathcal{D}(X)$ in $\$ 2.5$ namely

$$
\mathcal{D}(X) \simeq \mathrm{SU}(2) \times_{\mathbb{Z} / 2 \mathbb{Z}} \Gamma\left(S^{2} ; \mathrm{SO}(3)\right) .
$$

When $\operatorname{genus}(\Sigma)>0$ the fibration $X \rightarrow \Sigma$ is pulled back from $\mathbb{C} P^{2} \# \overline{\mathbb{C P}}^{2} \rightarrow S^{2}$, and it is not hard to see that elements in all components of $\operatorname{Diff}^{+}(\Sigma)$ lift. Moreover, one can check that

$$
\mathcal{D}(X) \simeq \operatorname{Diff}^{+}(\Sigma) \times \Gamma(\Sigma ; \mathrm{SO}(3))
$$

One can also consider the other limit, when the relative size of the base goes to zero. The case $X=S^{2} \times S^{2}$ is exceptional, since there is an extra symmetry of $X$ that interchanges the base and fiber. For all other $X$ the fiber class $F$ is distinguished by the fact that every tame $J$ defines a fibering of $X$ by $J$-holomorphic spheres in class $F$. When $X=\mathbb{C} P^{2} \# \overline{\mathbb{C}}^{2}$ the limit that we are now considering is given by $\lambda \rightarrow-1$, and the groups $G_{\lambda}$ stabilize: by Gromov's work they all equal $\mathrm{U}(2)$ as soon as $\lambda \leq 0$. It would be interesting to know if this result had analogs when $\operatorname{genus}(\Sigma)>0$.

\section{Relation With Kronheimer's WORK}

In [9], Kronheimer described some nontrivial families $\mathcal{F}_{X}$ of cohomologous symplectic structures on certain 4-manifolds $X$. In particular he showed how to construct a family $\mathcal{F}_{\ell}$ of symplectic forms $\omega^{\nu}$ on $S^{2} \times S^{2}$ in class $\left[\omega_{\lambda}\right]$ that are parametrized by $\nu \in S^{4 \ell+1}$. In many cases, he shows by an argument using Seiberg-Witten 
invariants that $\mathcal{F}_{X}$ represents a nontrivial homotopy class in the appropriate space $\mathcal{S}_{X}$ of cohomologous symplectic forms on $X$. Since his argument only works when $b^{+}(X)$ is sufficiently large, it does not apply directly to $S^{2} \times S^{2}$ or to $\mathbb{C} P^{2} \# \overline{\mathbb{C} P}^{2}$. In this section we exploit the close connection between $\mathcal{F}_{\ell}$ and $w_{\ell}$ to show that $\mathcal{F}_{\ell}$ is nontrivial also in these cases.

As was pointed out at the beginning of the introduction, both $M^{0}=S^{2} \times$ $S^{2}$ and $M^{1}=\mathbb{C} P^{2} \# \overline{\mathbb{C} P}^{2}$ have a unique symplectic structure in each permissible cohomology class. In particular, this means that the spaces

$$
\mathcal{S}_{\lambda}^{i} \equiv\left\{\text { all symplectic forms on } M^{i} \text { representing the cohomology class }\left[\omega_{\lambda}^{i}\right]\right\}
$$

are homogeneous for the groups $\operatorname{Diff}\left(M^{i}\right)$ of all diffeomorphisms of $M^{i}$ :

$$
\mathcal{S}_{\lambda}^{i} \cong \operatorname{Diff}\left(M^{i}\right) / G_{\lambda}^{i}, \quad i=0,1
$$

The corresponding fibration

$$
G_{\lambda}^{i} \longrightarrow \operatorname{Diff}\left(M^{i}\right) \longrightarrow \mathcal{S}_{\lambda}^{i}
$$

has an associated long exact sequence

$$
\cdots \rightarrow \pi_{k}\left(\operatorname{Diff}\left(M^{i}\right)\right) \rightarrow \pi_{k}\left(\mathcal{S}_{\lambda}^{i}\right) \stackrel{\partial}{\rightarrow} \pi_{k-1}\left(G_{\lambda}^{i}\right) \rightarrow \pi_{k-1}\left(\operatorname{Diff}\left(M^{i}\right)\right) \rightarrow \cdots
$$

Since the rational cohomology of any $H$-space is freely generated by its rational homotopy, Theorems 1.1 and 1.4 imply that $\pi_{*}\left(G_{\lambda}^{i}\right) \otimes \mathbb{Q}$ has four generators, in degrees $1,3,3$ and $4 \ell+2 i$. The first three of these map to nonzero elements in $\operatorname{Diff}\left(M^{i}\right)$, but the fourth does not since it already vanishes in $G_{\lambda+1}^{i}$. Hence it must lie in the image of the boundary map $\partial$ of the above long exact sequence. Thus we have:

Proposition 5.1. For $i=0,1$, and $0 \leq \ell-1<\lambda \leq \ell, \ell \in \mathbb{N}$, there exists a nonzero element $\mathcal{F}_{\lambda} \in \pi_{4 \ell+1+2 i}\left(\mathcal{S}_{\lambda}^{i}\right)$ whose image in $\pi_{4 \ell+2 i}\left(G_{\lambda}^{i}\right)$ maps to a nontrivial generator of $H_{4 \ell+2 i}\left(G_{\lambda}^{i}, \mathbb{Q}\right)$.

Remark 5.2. When $\ell=0$ (i.e. $\lambda=0$ for $i=0$, or $-1<\lambda \leq 0$ for $i=1$ ) the boundary map $\partial: \pi_{1+2 i}\left(\mathcal{S}_{\lambda}^{i}\right) \rightarrow \pi_{2 i}\left(G_{\lambda}^{i}\right)$ is zero.

Kronheimer gave an explicit construction for this element $\mathcal{F}_{\ell}$ at least in the case $\lambda=\ell$, which shows clearly why it vanishes when $\lambda$ increases past $\ell$. We will first describe this construction and then relate it to the picture given in Definition 3.10 of $w_{\ell}$. As usual, we restrict to the case $i=0$ and omit superscripts. The case $i=1$ is almost identical.

The construction goes as follows. Let $V$ be an open neighborhood of $\{0\} \in \mathbb{C}^{2 \ell+1}$. For $\ell \geq 1$ Kronheimer constructs in [9], §3, a smooth family

$$
\left(X^{v}, J^{v}, \tau_{\ell}^{v}\right), \quad v \in V-\{0\}
$$

of Kähler manifolds diffeomorphic to $X=S^{2} \times S^{2}$ with Kähler form $\tau_{\ell}^{v}$ in class $\left[\omega_{\ell}\right]$. This family can be completed by a complex fiber $\left(X^{0}, J^{0}\right)$ over 0 in such a way that the following conditions are satisfied.

(i) The whole family $\mathbf{X}=\bigcup_{v \in V} X^{v}$ is smoothly trivial, i.e. is fiberwise diffeomorphic to $X \times V$. Moreover there is a complex structure on $\mathbf{X}$ that restricts to $J^{v}$ on each fiber $X^{v}$, and a closed $(1,1)$ form $\tau_{\ell}$ on $\mathbf{X}$ that restricts to $\tau_{\ell}^{v}$ when $v \neq 0$.

(ii) The restriction of $\tau_{\ell}$ to $X^{0}$ degenerates along a holomorphic curve $\Delta$ in class $E_{2 \ell+2}=E_{0}-(\ell+1) F$. 
(iii) For any $\lambda>\ell$ there is a Kähler form $\tau_{\lambda}$ on $\mathbf{X}$ whose restriction $\tau_{\lambda}^{v}$ to each $X^{v}$ lies in the class $\left[\omega_{\lambda}\right]$. Moreover the family $\tau_{\mu}, \mu \geq \ell$, varies smoothly with $\mu$.

Condition (ii) implies that $\left(X^{0}, J^{0}\right)$ is simply $\left(S^{2} \times S^{2}, J_{2 \ell+2}\right)$. The complex variety $Y^{0}$ that is obtained from $X^{0}$ by collapsing the curve $\Delta$ has a quotient singularity of the form $\mathbb{C}^{2} / C_{2 \ell+2}$ where $C_{p}$ is the cyclic group of order $p$ acting diagonally. If $\mathbf{Y}$ is obtained from $\mathbf{X}$ by collapsing $\Delta, \mathbf{Y}$ is smooth and the form $\tau_{\ell}$ descends there. In fact, as is explained in [9], §5, $\mathbf{Y}$ is the Artin component of the universal unfolding of the given quotient singularity, and $\mathbf{X}$ is its simultaneous resolution.

Several points in this construction are relevant to us. First observe that we can consider the forms $\tau_{\lambda}^{v}$ to lie on the fixed manifold $X=S^{2} \times S^{2}$ by using the smooth trivialization given in (i). When $\lambda=\ell$ we therefore get a family $\tau_{\ell}^{\nu}, \nu \in V-\{0\}$, of Kähler forms on $X$ and hence an element $\mathcal{F}_{\ell}^{\prime} \in \pi_{4 \ell+1}\left(\mathcal{S}_{\ell}\right)$. We will show:

Proposition 5.3. $\partial\left(\mathcal{F}_{\ell}^{\prime}\right) \neq 0$, where $\partial: \pi_{*+1}\left(\mathcal{S}_{\ell}\right) \rightarrow \pi_{*}\left(G_{\ell}\right)$ is the boundary map considered above.

Thus we can take $\mathcal{F}_{\ell}=\mathcal{F}_{\ell}^{\prime}$ in Proposition 5.1 This picture explains why $\mathcal{F}_{\lambda}$ vanishes when $\lambda$ increases beyond $\ell$. For, as mentioned in (iii) above, the family $\tau_{\ell}^{v}, v \in V-\{0\}$, smoothly deforms to a family $\tau_{\lambda}^{v}, \lambda>\ell$, that extends to $v=0$. Using this, it is easy to see that if $G_{\ell} \rightarrow G_{\lambda}$ is constructed as in $\partial\left(\mathcal{F}_{\ell}\right)$ is in the kernel of the induced map on $\pi_{4 \ell}$.

As a first step in the proof of this proposition, observe that when $\lambda>\ell$ we can trivialize the family $\left(X^{v}, \tau_{\lambda}^{v}\right), v \in V$, as a family of symplectic manifolds, identifying them all with $\left(S^{2} \times S^{2}, \omega_{\lambda}\right)$. This gives us a family

$$
\mathcal{V}_{\lambda}=\left\{J^{\nu}: \nu \in V\right\}
$$

of elements of $\mathcal{J}_{\lambda}$. Condition (ii) implies that $J^{0}$ is an element in the stratum $U_{\ell+1}$ that we can identify with $J_{2 \ell+2}$. Moreover, as pointed out at the end of [9], $\S 4$, this family $\mathcal{V}_{\lambda}$ is transverse to $U_{\ell+1}$. In fact, since $U_{\ell+1}$ has (real) codimension precisely $4 \ell+2$ in $\mathcal{J}_{\lambda}, \mathcal{V}_{\lambda}$ is a normal slice to $U_{\ell+1}$. Therefore, we can investigate the structure of the links of one stratum in another by looking at the intersections of these strata with $\mathcal{V}_{\lambda}$. In particular, none of these intersections is empty and the boundary of the intersection $U_{\ell} \cap \mathcal{V}_{\lambda}$ can be identified with the link $L_{\ell+1}$.

Now, let us look at the corresponding picture when $\lambda=\ell$. In this case, we restrict $v$ to a small $(4 \ell+1)$-sphere $S_{V}$ that encloses $\{0\}$ in $V$ and pick a basepoint $* \in S_{V}$ at which $J^{*}$ is diffeomorphic to the standard split structure. Let $D$ be a neighborhood of $*$ in $V_{S}$ that is diffeomorphic to an open disc and is small enough that the $J^{v}$ for $v \in \bar{D}$ are all split. Then trivialize the symplectic manifolds $\left(X^{v}, \tau_{\ell}^{v}\right)$ for $v$ in the disk $V_{S}-D$, identifying them all with $\left(S^{2} \times S^{2}, \omega_{\ell}\right)$. This gives us a smooth family

$$
\mathcal{W}_{\ell}=\left\{J^{\nu}: \nu \in V_{S}-D\right\} \subset \mathcal{J}_{\ell}
$$

Moreover, our choice of $D$ implies that $\partial \mathcal{W}_{\ell}$ lies entirely in $U_{0}$. Thus we get an element $\beta_{\ell}$ of $\pi_{4 \ell}\left(U_{0}\right)$.

Lemma 5.4. This element $\beta_{\ell} \in \pi_{4 \ell}\left(U_{0}\right)$ lifts to $\partial\left(\mathcal{F}_{\ell}^{\prime}\right) \in \pi_{4 \ell}\left(G_{\ell}\right)$.

Proof. To see that $\beta_{\ell}$ lifts to $G_{\ell}$, we just need to perform the previous construction with a little more care. By construction, we can identify all the Kähler manifolds $\left(X^{v}, J^{v}, \tau_{\ell}^{v}\right), v \in \bar{D}$, with the standard model $\left(S^{2} \times S^{2}, J_{0}, \omega_{\ell}\right)$. Then the manifolds 
$\left(X^{v}, \tau_{\ell}^{v}\right), v \in \partial D$, have two identifications with $S^{2} \times S^{2}$ and the difference between these two gives the desired lift. This lift represents $\partial\left(\mathcal{F}_{\ell}^{\prime}\right)$ by the definition of the boundary map $\partial$.

QED

The next claim is that the sets

$$
\mathcal{W}_{\ell} \subset \mathcal{J}_{\ell} \quad \text { and } \quad \mathcal{V}_{\lambda} \subset \mathcal{J}_{\lambda}, \quad \lambda>\ell,
$$

fit together nicely as $\lambda$ varies. More precisely, define

$$
\mathcal{W}_{\lambda}=\left\{J^{\nu}: \nu \in S_{V}-D\right\}
$$

and, for some $\varepsilon>0$, consider the space

$$
\mathcal{W}=\bigcup_{\mu \in[\ell, \ell+\varepsilon)} \mathcal{W}_{\mu}
$$

Each space $\mathcal{W}_{\lambda}$ inherits a stratification from $\mathcal{J}_{\lambda}$, and it is not hard to see that by careful use of the Moser method one can construct all the symplectic trivializations to vary smoothly with $\mu$. Thus we have:

Lemma 5.5. The stratifications of each $\mathcal{W}_{\mu}$ fit together to give a stratification of $\mathcal{W}$ by the sets $U_{0} \cap \mathcal{W}, \ldots, U_{\ell} \cap \mathcal{W}$. In particular, the intersections $U_{k} \cap \mathcal{W}_{\mu}$ for $\mu=\ell$ and $\mu>\ell$ are diffeomorphic.

Corollary 5.6. The intersection $U_{\ell} \cap \mathcal{W}_{\ell}$ is a connected 3-dimensional set that represents a generator of $H_{3}\left(U_{\ell}\right)$.

Proof. By the previous lemma it suffices to prove this for $\mu>\ell$. But we remarked before that the boundary of the intersection $U_{\ell} \cap \mathcal{V}_{\lambda}$ can be identified with the link $L_{\ell+1}$. Now use Corollary 3.2 .

QED

Remark 5.7. One can construct $\mathcal{W}_{\ell}$ so that it is invariant under the action of the $\mathrm{SU}(2)$ factor in the automorphism group $K_{\ell+1}$ of $J_{2 \ell+2}$. Then the intersection $U_{\ell} \cap$ $\mathcal{W}_{\ell}$ is just an $\mathrm{SU}(2)$-orbit and so, by Lemma 2.10, represents the class $\xi+(\ell+1)^{2} \eta$.

The proof of Proposition 5.3 is completed by the following result.

Proposition 5.8. The image $v_{0}$ of $w_{\ell}$ in $H^{4 \ell}\left(U_{0}\right)$ does not vanish on $\beta_{\ell}$.

Proof. $\mathcal{W}_{\ell}$ is a $(4 \ell+1)$-dimensional disk in $\mathcal{J}_{\ell}$ that intersects all the strata $U_{k}$ transversally. We have just seen that it intersects $U_{\ell}$ in a cycle $C$ that generates $H_{3}\left(U_{\ell}\right)$. Let $Z \subset \mathcal{W}_{\ell}$ be the boundary of a tubular neighborhood $\mathcal{N}(C)$ of $C$. Then, $Z$ has dimension $4 \ell$ and lies in $\left(U_{0 \ldots \ell-1}\right) \cap \mathcal{W}_{\ell}$. In the notation of Remark 3.11 $Z=C * D$, where in fact $C=\xi+(\ell+1)^{2} \eta$ (though this will not be needed). Thus $\widehat{y}_{\ell}(Z) \neq 0$. Further, since $C=\mathcal{W}_{\ell} \cap U_{\ell}$,

$$
\mathcal{W}_{\ell}-\mathcal{N}(C) \subset U_{0 \ldots \ell-1} .
$$

Since $C$ is connected, $H^{4 \ell-4}\left(\mathcal{W}_{\ell}-\mathcal{N}(C)\right)=\mathbb{Q}$. Hence the cycle $Z-\beta_{\ell}$ is null homologous in $\mathcal{W}_{\ell}-\mathcal{N}(C)$ and hence in $U_{0 \ldots \ell-1}$. Thus

$$
v_{0}\left(\beta_{\ell}\right)=\widehat{y}_{\ell}\left(\beta_{\ell}\right)=\widehat{y}_{\ell}(Z) \neq 0,
$$

as claimed. 


\section{Whitehead PRoducts AND $H^{*}\left(B G_{\lambda}\right)$}

Here we exhibit the $w_{\ell}$ as dual to certain higher products in the Lie algebra $\pi_{*}\left(G_{\lambda}\right) \otimes \mathbb{Q}$. Thus they are desuspensions of higher Whitehead products in $\pi_{*}\left(B G_{\lambda}\right)$, and hence, via the work of Andrews-Arkowitz 4], give rise to relations in the rational cohomology of the classifying spaces $H^{*}\left(B G_{\lambda}\right)$. In fact, our knowledge of $H^{*}\left(G_{\lambda}\right)$ allows us to calculate the differential graded Lie algebra $\pi_{*-1}\left(B G_{\lambda}\right) \otimes \mathbb{Q}$ and hence to determine the rational homotopy type of $B G_{\lambda} \sqrt{2}$ In particular, since $H^{*}\left(B G_{\lambda}\right)$ is not a free ring, $B G_{\lambda}$ does not have the rational homotopy type of an $H$-space.

First recall (see for example Ch. X of [18]) that for any group $G$ the Samelson product $[\alpha, \beta] \in \pi_{p+q}(G)$ of elements $\alpha \in \pi_{p}(G)$ and $\beta \in \pi_{q}(G)$ is represented by the map

$$
S^{p+q}=S^{p} \times S^{q} / S^{p} \vee S^{q} \longrightarrow X:(u, v) \mapsto \alpha(u) \beta(v) \alpha(u)^{-1} \beta(v)^{-1} .
$$

(For simplicity of notation, we will use the same letter for a map $\tau: C \rightarrow X$ as for the element of homotopy or homology that it represents.) The Samelson product in $\pi_{*}(G)$ is related to the Pontrjagin product $*$ in $H_{*}(G, \mathbb{Z})$ by the formula

$$
[\alpha, \beta]=\alpha * \beta-(-1)^{p q} \beta * \alpha \in H_{*}(G) .
$$

Here we have suppressed mention of the Hurewicz map $\pi_{*}(G) \rightarrow H_{*}(G)$, and have written $\alpha * \beta$ for the cycle

$$
S^{p} \times S^{q} \rightarrow G: \quad(u, v) \mapsto \alpha(u) \cdot \beta(v) .
$$

Thus the domain $\operatorname{Dom}(\alpha * \beta)$ of $\alpha * \beta$ is $S^{p} \times S^{q}$, while $\operatorname{Dom}(\beta * \alpha)$ is $S^{q} \times S^{p}$. Moreover, if we think of $\alpha, \beta \in \pi_{*}(G)$ as the desuspensions of elements $E(\alpha), E(\beta)$ in $\pi_{*}(B G)$, the Samelson product $[\alpha, \beta]$ is (up to sign) the desuspension of the Whitehead product $[E(\alpha), E(\beta)] \in \pi_{p+q+1}(B G)$; see [18], Thm. (X.7.10). Hence the Samelson products in $G$ vanish if $B G$ itself is an $H$-space, since Whitehead products vanish in $H$-spaces.

We will say that $\alpha, \beta$ are commuting representatives for their respective classes if

$$
\alpha(u) \beta(v)=\beta(v) \alpha(u) \quad \text { for all } \quad u, v \in \operatorname{Dom}(\alpha) \times \operatorname{Dom}(\beta) .
$$

Thus, in this case, $[\alpha, \beta]$ is trivially 0 .

Let us first consider the case $i=0$ and $0<\lambda \leq 1$. As usual we suppress the superscript $i$ and write $w$ instead of $w_{\ell}$. We will also write $G$ instead of $G_{\lambda}$ and will work with rational coefficients. Further, let $\alpha=\alpha_{1}: S^{1} \rightarrow G$ and $\xi=\xi_{0}: S^{3} \rightarrow G$ be as in 2.5 . Note that $[\xi, \eta]=0$ because $\xi, \eta$ have commuting representatives in $K_{0}$, while $[\xi, \xi]$ and $[\eta, \eta]$ vanish because $\pi_{6}\left(S^{3}\right)$ is finite.

Lemma 6.1. For $0<\lambda \leq 1,[\alpha, \xi+\eta]=0$ while $w([\alpha, \xi]) \neq 0$.

Proof. By Lemma 2.10 the classes $\alpha$ and $\xi+\eta$ have commuting representatives in $K_{1} \subset G=G_{\lambda}$, which proves the first statement.

To prove the second, note first that because $\xi$ has image in $K_{0}$, it maps to a trivial element in $U_{0}=G / K_{0}$. Hence the image of $[\alpha, \xi]=\alpha * \xi+\xi * \alpha$ in $H_{4}\left(U_{0}\right)$ is simply the Pontrjagin product $\xi * \alpha$. Now $G$ maps to $U_{0}$ via $\phi \mapsto \phi_{*}(J)$ where we can choose any $J \in U_{0}$. If we choose $J \in \mathcal{N}\left(U_{1}\right)$ very close to $J_{1} \in U_{1}$, then the

\footnotetext{
${ }^{2}$ The calculations in $\$ 3$ show that $G_{\lambda}$, and hence $B G_{\lambda}$, has finite rational homotopy type. Moreover $\pi_{1}\left(B G_{\lambda}\right) \otimes \mathbb{Q}=0$. Thus the work of [4] applies.
} 
loop $\theta \mapsto \alpha(\theta)_{*}(J)$ circles the stratum $U_{1}$ while the sphere $u \mapsto \xi(u)_{*}(J)$ is a copy in $\mathcal{N}\left(U_{1}\right)-U_{1}$ of a generating cycle for $H_{3}\left(U_{1}\right)$. Thus the map

$$
H_{*}\left(\partial \mathcal{N}\left(U_{1}\right)\right) \rightarrow H_{*-1}\left(U_{1}\right)
$$

induced by integration over the fiber takes the Pontrjagin product $\xi * \alpha$ to a generator of $H_{3}\left(U_{1}\right)$. Since this map is dual to the map $\tau$ occurring in $\left(M V_{1}\right)$ it follows from Definition 3.10 that the element $v_{0} \in H^{4}\left(U_{0}\right)$ that corresponds to $w$ takes a nonzero value on $\xi * \alpha$.

QED

Corollary 6.2. When $0<\lambda \leq 1, H^{*}\left(B G_{\lambda}\right)=\mathbb{Q}[A, X, Y] /\{A(X-Y)=0\}$, where $A$ has dimension 2 and $X, Y$ have dimension 4.

Proof. The structure theorem for the rational cohomology of an $H$-space $G$ states that $H^{*}(G)$ is freely generated as a graded algebra by the rational homotopy groups $\pi_{*}(G) \otimes \mathbb{Q}$. Thus $\pi_{*}(G) \otimes \mathbb{Q}=0$ for $* \neq 1,3,4$, and is generated by the elements $\alpha, \xi, \eta,[\alpha, \xi]$. Since $[\alpha, \xi+\eta]=0$, the only nontrivial Whitehead product in $B G$ (including higher order ones) is the surjective map

$$
[\cdot, \cdot]: \pi_{2}(B G) \otimes \pi_{4}(B G) \otimes \mathbb{Q} \rightarrow \pi_{5}(B G) \otimes \mathbb{Q}=\mathbb{Q}
$$

with kernel $E(\alpha) \otimes \mathbb{Q}(E(\xi+\eta))$. By [4], Theorem 5.4, the dual of this map corresponds to a relation in $H^{*}(B G)$.

More precisely, when one constructs the minimal model $\mathcal{M}$ of $H^{*}(B G)$, there is a generator $A \in \mathcal{M}^{2}$ dual to $E(\alpha)$, generators $X$ and $Y$ in $\mathcal{M}^{4}$ dual to $E(\xi)$ and $E(\eta)$, and a generator $W$ in $\mathcal{M}^{5}$ corresponding to $E([\alpha, \xi])$. The image $d W$ of $W$ under the differential $d: \mathcal{M}^{5} \rightarrow \mathcal{M}^{6}$ is dual to the above Whitehead product map $[\cdot, \cdot]$. Hence $d W=A(X-Y)$.

Moreover, if $\mathcal{A}$ denotes the vector space of indecomposable (or primitive) elements in $\mathcal{M}$ and $\mathcal{M}_{\geq k}$ is the subalgebra generated by $\mathcal{A}^{\otimes k}$, the differential $d$ : $\mathcal{M} \rightarrow \mathcal{M} / \mathcal{M}_{\geq k+2}$ is determined by the nonvanishing Whitehead products of order $\leq k$. In the case of $B G_{\lambda}$ for $0<\lambda \leq 1$ it is only the first order, i.e. the usual, Whitehead products, that can be nonzero, since these account for the whole of $\pi_{*}(G)$. It follows that $H^{*}(B G)$ has only the one relation $A(X-Y)=0$. QED

Remark 6.3. Another way of seeing the relation between Samelson (or Whitehead) products and $H^{*}(B G)$ is to look at the cohomology spectral sequence of the fibration $G \rightarrow E G \rightarrow B G$. In general, the relation is complicated, and the more natural spectral sequence to look at is one constructed by Quillen; see Allday [2]. However, in the case considered above it is not too hard to work out what happens since only first order products are involved. The differential

$$
d_{2}^{0, q}: H^{0}(B G) \otimes H^{q}(G) \rightarrow H^{2}(B G) \otimes H^{q-1}(G)
$$

takes $a$ to $A$ and $x, y$ to 0 , and, when $q=4$, its restriction to the primitive part of $H^{4}(G)$ is dual to the Samelson product

$$
H_{2}(B G) \otimes H_{3}(G) \cong \pi_{1}(G) \otimes \pi_{3}(G) \otimes \mathbb{Q} \rightarrow \pi_{4}(G) \otimes \mathbb{Q} .
$$

Thus

$$
d_{2}^{0,4}(w)=A \otimes(x-y)=d_{2}(a \cup(x-y)),
$$

so that

$$
E_{3}^{0,4} \cong E_{4}^{0,4} \cong \mathbb{Q} \widehat{w}
$$


Similarly,

$$
d_{4}^{0,4}: H^{0}(B G) \otimes H^{4}(G) \rightarrow H^{4}(B G) \otimes H^{1}(G)
$$

is dual to the Samelson product and so takes the generator $\widehat{w}$ of $E_{4}^{0,4}$ to the element $a(X-Y)$ of

$$
E_{4}^{4,1}=\operatorname{Ker}\left(d_{2}^{4,1}\right) \subset H^{4}(B G) \otimes H^{1}(G) .
$$

Thus $a(X-Y)$ must lie in the kernel of $d_{2}^{4,1}$, i.e. $A(X-Y)=0$ in $H^{*}(B G)$.

Next, let us consider the case $1<\lambda \leq 2$, i.e. $\ell=2$. We will discuss this case in detail since it is a paradigm for the others. Now, the first order Samelson products $[\alpha, \xi],[\alpha, \eta]$ both vanish since $[\alpha, \xi+\eta]=0$ as before, and the classes $\alpha$ and $\xi+4 \eta$ have commuting representatives in $K_{2}$. Hence all first order Samelson products vanish. Therefore, the second order Samelson product

$$
[\alpha, \xi, \xi] \in \pi_{8}(G) \otimes \mathbb{Q}
$$

is defined. As explained in [2, in general $[\alpha, \xi, \xi]$ should be considered as a coset in $\pi_{8}(G) \otimes \mathbb{Q}$, but this is reduced to a single element because the relevant homotopy groups vanish. More precisely, given two maps $\beta_{i}: S^{p_{i}} \rightarrow X, i=1,2$, whose Samelson product

$$
\left[\beta_{1}, \beta_{2}\right]: S^{p_{1}+p_{2}} \rightarrow X
$$

is null-homotopic, let us write $C\left[\beta_{1}, \beta_{2}\right]$ for the chain given by a particular choice of null-homotopy $D^{p_{1}+p_{2}+1} \rightarrow X$. (This is unique up to homotopy.) Then, Allday shows in [3], $\S 2$, that the image of $[\alpha, \xi, \xi]$ in $H_{8}(G)$ is represented by

$$
[\alpha, \xi, \xi]=[C[\alpha, \xi], \xi]+[C[\xi, \xi], \alpha]+[C[\xi, \alpha], \xi]=2[C[\alpha, \xi], \xi]+[C[\xi, \xi], \alpha],
$$

since $[\alpha, \xi]=[\xi, \alpha]$. This is a cycle because of the Jacobi identity. Its suspension is the obstruction to extending the map

$$
E(\alpha) \vee E(\xi) \vee E(\xi): \quad S^{2} \vee S^{4} \vee S^{4} \longrightarrow B G
$$

to the product $S^{2} \times S^{4} \times S^{4}$.

Lemma 6.4. When $\ell=2$,

(i) $[\alpha, \xi+\eta, \xi+\eta]=[\alpha, \xi+4 \eta, \xi+4 \eta]=0$.

(ii) $w_{2}([\alpha, \xi, \xi]) \neq 0$.

Proof. First observe that when $k=1$ or 2, one can choose commuting representatives for $\alpha$ and $\xi+k^{2} \eta$ in $K_{k}$. Moreover, one can also choose a representative for $C\left[\xi+k^{2} \eta, \xi+k^{2} \eta\right]$ that lies in the $\mathrm{SO}(3)$ factor of $K_{k}$ and so commutes with $\alpha$. This proves (i).

Now consider (ii). As in Lemma 6.1, let us look at the image of this cycle in $U_{0}$. Since $[\xi, \xi]=0$ in $H_{*}\left(K_{0}\right)$ we can choose $C[\xi, \xi]$ to lie in $K_{0}$. Therefore, in $U_{0}=G / K_{0}$ the class $[\alpha, \xi, \xi]$ is represented by

$$
2 \xi * C[\alpha, \xi]+C[\xi, \xi] * \alpha .
$$

Note that $G$ acts on $\mathcal{J}=\mathcal{J}_{\lambda}$ by $J \mapsto \phi_{*}(J)$, and we may choose the neighborhood $\mathcal{N}\left(U_{1}\right)$ to be invariant under the induced action of the compact group $K_{0}$. Further, given a $K_{0}$ action $K_{0} \times V \rightarrow V$, we write $*$ to denote the induced product

$$
*: \quad H_{i}\left(K_{0}\right) \otimes H_{j}(V) \rightarrow H_{i+j}(V) .
$$


If we choose $J \in \mathcal{N}\left(U_{1}\right)-U_{1}$ as in Lemma 6.1, the chains $C[\xi, \xi], \alpha$, and $C[\xi, \xi] * \alpha$ all have representatives in $\mathcal{N}\left(U_{1}\right)-U_{1}$. In particular, $C[\xi, \xi] * \alpha$ can be considered as an element of $H_{8}\left(\mathcal{N}\left(U_{1}\right), \partial \mathcal{N}\left(U_{1}\right)\right)$ with boundary $-2 \xi *[\alpha, \xi] \in H_{7}\left(\partial \mathcal{N}\left(U_{1}\right)\right)$. But

$$
H_{8}\left(\mathcal{N}\left(U_{1}\right), \partial \mathcal{N}\left(U_{1}\right)\right) \cong H_{4}\left(U_{1}\right)=0 .
$$

Thus we can replace the chain $C[\xi, \xi] * \alpha$ (which does not intersect $U_{1}$ ) by any chain with the same boundary. But there is an obvious chain in $\mathcal{N}\left(U_{1}\right)$ with boundary $[\alpha, \xi]=\xi * \alpha$, namely, $\xi * D$ where $D$ is a 2 -disc in $\mathcal{N}\left(U_{1}\right)$ that is transverse to the stratum $U_{1}$. Thus $[\alpha, \xi, \xi]$ is represented by

$$
\xi *(C[\alpha, \xi]-2 \xi * D),
$$

where $C[\alpha, \xi]-2 \xi * D$ is a 5 -cycle in $U_{01}$. Observe that $D$ has nontrivial intersection with $U_{1}$ while $C[\alpha, \xi]$ lies in $U_{0}$. Hence the cycle $C[\alpha, \xi]-2 \xi * D$ has nontrivial linking number with $U_{2}$. In other words, its image under the map $\tau: H^{5}\left(U_{01}\right) \rightarrow H^{0}\left(U_{2}\right)$ of $\left(M V_{2}\right)$ is nonzero. The desired conclusion now follows from Definition 3.10 QED

Corollary 6.5. When $1<\lambda \leq 2$,

$$
H^{*}\left(B G_{\lambda}\right)=\mathbb{Q}[A, X, Y] /\{A(X-Y)(4 X-Y)=0\},
$$

where $A$ has dimension 2 and $X, Y$ have dimension 4.

Proof. Again, the relations in $H^{*}(B G)$ are dual to the nonzero higher Whitehead products in $\pi_{*}(B G)$; see [4], Thm. 5.4. Since $[\alpha, \alpha]=0$, the three elements $\alpha, \xi, \eta$ are transgressive and give rise to nonzero elements $A, X, Y$ of $H^{*}\left(B G_{\lambda}\right)$. Moreover, since only one of the groups $\pi_{*}(B G) \otimes \mathbb{Q}=\pi_{*-1} G \otimes \mathbb{Q}$ for $*>4$ is nonzero, there can be at most one relation between these generators. In fact, the question here is to decide whether the new element $w_{\ell}$ is transgressive in the spectral sequence of the fibration $G \rightarrow E G \rightarrow B G$, i.e. gives rise to a new generator of $H^{*}(B G)$, or whether it gives rise to a relation between the existing generators. The previous lemma tells us that the latter holds. Thus there is exactly one nontrivial relation $F=0$ in $H^{*}(B G)$. Since it corresponds to a second order Whitehead product, $F$ is homogeneous of order 3 in the variables $A, X, Y$, and we can think of it as a symmetric trilinear function on the vector space $V$ that is spanned over $\mathbb{Q}$ by a basis $\left\{e_{\alpha}, e_{\xi}, e_{\eta}\right\}$ dual to $\{A, X, Y\}$. The vanishing results in Lemma 2.10 (i) tell us that

$$
F\left(e_{\alpha}, e_{\xi}+e_{\eta}, e_{\xi}+e_{\eta}\right)=0, \quad F\left(e_{\alpha}, e_{\xi}+4 e_{\eta}, e_{\xi}+4 e_{\eta}\right)=0 .
$$

Since $F\left(e_{\alpha}, e_{\xi}, e_{\xi}\right) \neq 0, F$ has to be a nonzero multiple of $A(X-Y)(4 X-Y)$, as claimed.

QED

When $\ell>2$ the argument is similar. We first explain higher order Whitehead products. Suppose maps $\beta_{i}: S^{p_{i}} \rightarrow X$ are given such that

$$
\beta_{1} \vee \cdots \vee \beta_{k}: \quad S^{p_{1}} \vee \cdots \vee S^{p_{k}} \longrightarrow X
$$

extends to a map $f$ of the $(p-1)$-skeleton $T_{I}$ of $P_{I}=S^{p_{1}} \times \cdots \times S^{p_{k}}$ into $X$, where $p=\sum_{i} p_{i}$ and $I=\left(p_{1}, \ldots, p_{k}\right)$. Then the usual higher Whitehead product $\left[\beta_{1}, \ldots \beta_{k}\right]$ is defined to be the set of all obstructions $O b(f)$ to extending $f$ over $P_{I}$, where $f$ ranges over all possible extensions to $T_{I}$. Thus this product is a coset in $\pi_{p-1}(X) \otimes \mathbb{Q}$. In our situation the space $X$ corresponds to $B G$ and we must consider the analogous higher order products in $G$. These are also called higher Whitehead 
products (though it might be more logical to call them higher Samelson products). As usual the $p$ th order products are defined only when all relevant lower order ones vanish. According to [3], $\S 3$, they can be defined inductively as follows. Again, we will be somewhat sloppy with notation, and will consider the formulas below to define representatives of the given classes, as well as the classes themselves.

Suppose given representatives $\xi_{i}$ of elements $\xi_{i} \in \pi_{n_{i}}(G) \otimes \mathbb{Q}$, and let $I$ be a proper subset $\left\{j_{0}, \ldots, j_{k}\right\}$ of $I_{n}=\{0,1,2, \ldots, n\}$. Then the $k$ th order Whitehead product

$$
\nu_{I}=\left[\xi_{j_{0}}, \ldots, \xi_{j_{k}}\right] \in \pi_{N_{I}}(G) \otimes \mathbb{Q}
$$

of the elements $\xi_{j_{0}}, \ldots, \xi_{j_{k}}$ has degree $N_{I}=k+\sum_{j \in I} n_{j}$ if it is defined. If $\nu_{I}$ is nullhomotopic, then the corresponding map from the $N_{I}$-skeleton $T_{I}$ of the product of spheres

$$
P_{I}=S^{n_{1}+1} \times \cdots \times S^{n_{k}+1}
$$

extends to $P_{I}$ and, as above, we write $C \nu_{I}$ for this extension. We should think of $C \nu_{I}$ as a singular chain with boundary $\nu_{I}$. (Usually it depends on choices, though in our situation it will not.) We extend this definition to singleton sets $I=\{j\}$, by setting $C \nu_{I}=\xi_{j}$ in this case.

Now suppose that these chains $C \nu_{I}$ are defined for all proper subsets $I$ of $I_{n}=$ $\{0,1, \ldots, n\}$. In this situation Allday shows that a representative for the $n$th order product is given by a formula of the type

$$
\nu_{I_{n}}=\left[\xi_{0}, \ldots, \xi_{n}\right]=\sum_{I \subset I_{n}} \pm\left[C \nu_{I}, C \nu_{I_{n}-I}\right],
$$

where the sign depends on the shuffle $\left(I, I_{n}-I\right) \cdot 3$

In our situation, all intermediate homotopy groups vanish and so the $n$th order product $\zeta_{n}=[\alpha, \xi, \ldots, \xi]$ represents a single element of $\pi_{4 n}(G) \otimes \mathbb{Q}$. Moreover, it is determined in terms of the $\zeta_{k}$ for $0 \leq k<n$ and in terms of the $p$ th order products $\xi_{p}=[\xi, \ldots, \xi]$ which we can consider as (null homologous) cycles in $K_{0}$. (Here we take $\zeta_{0}=\alpha$.) Moreover,

$$
\zeta_{n}=[\alpha, \xi, \ldots, \xi]=\ell\left[\xi, C \zeta_{n-1}\right]+\text { lower order terms, }
$$

where by lower order terms we mean terms of the form $\left[C \xi_{p}, C \zeta_{n-p}\right]$ with $p>1$.

In the next lemma we think of $\xi_{p}, C \xi_{p}$ as chains in $K_{0}$ that, as in Lemma 6.4 act via the Pontrjagin product $*$ on chains in $\mathcal{J}_{\lambda}$. In particular, we denote by $S$ the link of $U_{\ell}$ in $\mathcal{J}_{\lambda}$. Thus $S \cong S^{4 \ell-3}$ and $\xi * S$ is a cycle in $U_{0 \ldots \ell-1}$. By Remark 3.11, this cycle generates $H_{4 \ell}\left(U_{0 \ldots \ell-1}\right) \cong H_{4 \ell}\left(U_{0}\right)$. Hence the next lemma implies immediately that $w_{\ell}\left(\zeta_{\ell}\right) \neq 0$.

Lemma 6.6. If $\ell-1<\lambda \leq \ell$, the $\ell$ th order Whitehead product $\zeta_{\ell} \in \pi_{4 \ell}\left(G_{\lambda}\right) \otimes \mathbb{Q}$ is defined and its image in $U_{0 \ldots \ell-1} \subset \mathcal{J}_{\lambda}$ may be represented by a nonzero multiple of the cycle $\xi * S$ described above.

Proof. We prove this by induction on $\ell$. It is true when $\ell=1,2$ by our previous work. To prove it for $\ell>2$ we start, as in Lemma 6.4 from the equation

$$
\zeta_{\ell}=[\alpha, \xi, \ldots, \xi]=\ell\left[\xi, C \zeta_{\ell-1}\right]+\text { lower order terms. }
$$

\footnotetext{
${ }^{3}$ In fact, Allday interprets this formula in the minimal model of $X=B G$, showing that it defines a primitive element there. Therefore it is spherical and can be considered as an element in $\pi_{*}(B G) \otimes \mathbb{Q}=\pi_{*-1}(G) \otimes \mathbb{Q}$.
} 
Since $\xi_{p}$ and $C \xi_{p}$ lie in $K_{0}$ the image of $\zeta_{\ell}$ in $U_{0}=G / K_{0}$ has the form

$$
\ell \xi * C \zeta_{\ell-1}+\text { terms of the form } C \xi_{p} * C \zeta_{\ell-p} \text {. }
$$

Again, the boundary of $\xi * C \zeta_{\ell-1}$ is $\xi * \zeta_{\ell-1}$. By the inductive hypothesis, the cycle $\zeta_{\ell-1}$ can be represented in a deleted neighborhood $\mathcal{N}\left(U_{\ell-1}\right)-U_{\ell-1}$ of $U_{\ell-1}$. Moreover, if we choose this neighborhood to be $K_{0}$-invariant, we can represent $\xi * \zeta_{\ell-1}$ and all the cycles $C \xi_{p} * C \zeta_{\ell-p}, p>1$, in the above formula there as well. Note, however, that $C \zeta_{\ell-1}$ cannot be represented there since, by the inductive hypothesis $\zeta_{\ell-1}=\xi * S$, which is clearly nonzero in $H_{*}\left(\partial \mathcal{N}\left(U_{\ell-1}\right)\right)$. On the other hand, $S$ is bounded by a ball $D$ in the full neighborhood $\mathcal{N}\left(U_{\ell-1}\right)$ so that $\zeta_{\ell-1}$ is the boundary of $\xi * D$ in $\mathcal{N}\left(U_{\ell-1}\right)$. Therefore, as in Lemma 6.4 we can replace the sum of terms $C \xi_{p} * C \zeta_{\ell-p}$ in equation (4) by a suitable multiple of $\xi * D$. The result now follows as before.

QED

Corollary 6.7. When $\ell-1<\lambda \leq \ell$,

$$
H^{*}\left(B G_{\lambda}\right)=\mathbb{Q}[A, X, Y] /\left\{A(X-Y)(4 X-Y) \cdots\left(\ell^{2} X-Y\right)=0\right\},
$$

where $A$ has dimension 2 and $X, Y$ have dimension 4.

Proof. By Lemma 2.10 all $\ell$ th order Whitehead products of the form

$$
\left[\alpha, \xi+k^{2} \eta, \ldots, \xi+k^{2} \eta\right], \quad 1 \leq k \leq \ell,
$$

vanish in $\pi_{*}\left(G_{\lambda}\right)$. The rest of the proof is much the same as the proof of Corollary 6.5 and is left to the reader.

QED

This proves Theorem 1.2, The proof of the corresponding result for $\mathbb{C} P^{2} \# \overline{\mathbb{C P}}^{2}$ will, as usual, be left to the reader.

\section{ACKNOWLEDGEMENTS}

Part of this work was carried out while the first author was a postdoctoral member of the School of Mathematics of the Institute for Advanced Study. He takes this opportunity to thank the staff and faculty of the Institute for providing a wonderful environment, both for him and his family. The second author would like to thank Allday for bringing the references [2, 3, 4] to her attention.

\section{REFERENCES}

[1] M. Abreu, Topology of symplectomorphism groups of $S^{2} \times S^{2}$, Inv. Math., 131 (1998), 1-23. MR 99k:57065

[2] C. Allday, Rational Whitehead products and a spectral sequence of Quillen, Pac. Journ. Math., 46 (1973), 313-323. MR 48:12519

[3] C. Allday, Rational Whitehead products and a spectral sequence of Quillen, II, Houston J. Math., 3 (1977), 301-308. MR 57:13935

[4] P. Andrews and M. Arkowitz, Sullivan's minimal models and higher order Whitehead products, Can. J. Math. XXX (1978), 961-982. MR 80b:55008

[5] M. Audin, The topology of torus actions on symplectic manifolds, Progress in Math. 93, Birkhäuser, 1991. MR 92m:57046

[6] M. Audin and F. Lafontaine (eds.), Holomorphic Curves in Symplectic Geometry, Progress in Math. 117, Birkhäuser, 1994. MR 95i:58005]

[7] M. Gromov, Pseudo holomorphic curves in symplectic manifolds, Inv. Math., 82 (1985), 307-347. MR 87j:53053

[8] P. Iglesias, Les $S O(3)$-variétés symplectiques et leur classification en dimension 4, Bull. Soc. Math. France, 119 (1991), 371-396. MR 92i:57023

[9] P. Kronheimer, Some non-trivial families of symplectic structures, Harvard preprint, 1998. 
[10] F. Lalonde and D. McDuff, $J$-holomorphic spheres and the classification of rational and ruled symplectic 4-manifolds, in Contact and Symplectic Geometry, ed. C.Thomas, Cambridge University Press (1996). MR 98d:57045

[11] D. McDuff, From symplectic deformation to isotopy, in Topics in Symplectic Topology (Irvine, CA 1996), Internat. Press, Cambridge MA.

[12] D. McDuff, Almost complex structures on $S^{2} \times S^{2}$, Duke. Math. Journal, 101 (2000), 135177. CMP 2000:07

[13] D. McDuff, Symplectomorphism groups of ruled surfaces, in preparation.

[14] D. McDuff and D.A. Salamon, Introduction to Symplectic Topology, 2nd edition, Oxford University Press, 1998. MR 2000g:53098

[15] D. McDuff and D.A. Salamon, J-holomorphic curves and quantum cohomology, University Lecture Series 6, American Mathematical Society, Providence, RI, 1994. MR 95g:58026

[16] D. Salamon, Seiberg-Witten invariants of mapping tori, symplectic fixed points and Lefschetz numbers, Proceedings of 5th Gökova Topology conference, Turkish Journal of Mathematics, (1998), 1-27.

[17] P. Seidel, Floer homology and the symplectic isotopy problem, D. Phil. thesis, Oxford (1997).

[18] G.W. Whitehead, Elements of Homotopy Theory, Graduate Texts in Math. 61, SpringerVerlag (1978). MR 80b:55001

Department of Mathematics, Instituto Superior Técnico, 1049-001 Lisbon, Portugal

E-mail address: mabreu@math.ist.utl.pt

Department of Mathematics, State University of New York at Stony Brook, Stony Brook, NeW York 11794-3651

E-mail address: dusa@math.sunysb.edu 